\title{
ARTICLE
}

\section{OTUB1 enhances TGF $\beta$ signalling by inhibiting the ubiquitylation and degradation of active SMAD2/3}

Lina Herhaus ${ }^{1}$, Mazin Al-Salihi', Thomas Macartney ${ }^{1}$, Simone Weidlich ${ }^{1}$ \& Gopal P. Sapkota ${ }^{1}$

SMAD transcription factors are key intracellular transducers of TGF $\beta$ cytokines. SMADs are tightly regulated to ensure balanced cellular responses to TGF $\beta$ signals. Ubiquitylation has a key role in regulating SMAD stability and activity. Several E3 ubiquitin ligases that regulate the turnover of SMADs are known; however, proteins that prevent the ubiquitylation or cause deubiquitylation of active SMADs remain undefined. Here we demonstrate that OTUB1 is recruited to the active phospho-SMAD2/3 complex only on TGF $\beta$ induction. Further, OTUB1 has a crucial role in TGF $\beta$-mediated gene transcription and cellular migration. OTUB1 inhibits the ubiquitylation of phospho-SMAD2/3 by binding to and inhibiting the E2 ubiquitinconjugating enzymes independent of its catalytic activity. Consequently, depletion of OTUB1 in cells causes a rapid loss in levels of TGF $\beta$-induced phospho-SMAD2/3, which is rescued by the proteasomal inhibitor bortezomib. Our findings uncover a signal-induced phosphorylation-dependent recruitment of OTUB1 to its target in the TGF $\beta$ pathway.

\footnotetext{
${ }^{1}$ MRC Protein Phosphorylation Unit, College of Life Sciences, University of Dundee, Dow Street, Dundee DD1 5 EH, Scotland, UK. Correspondence and requests for materials should be addressed to G.P.S. (email: g.sapkota@dundee.ac.uk).
} 
T he signalling pathways triggered by the transforming growth factor- $\beta$ (TGF $\beta$ ) family of cytokines control a plethora of cellular functions during embryogenesis and in adult tissues, including differentiation, proliferation, survival, extracellular matrix production and migration. Consequently, abnormal TGF $\beta$ signalling is associated with multiple human diseases such as fibrosis, immune disorders and cancer ${ }^{1-4}$. TGF $\beta$ signalling is initiated on ligand binding to a pair of receptor serine/threonine protein kinases (termed type II and type I) on the cell surface. This triggers the phosphorylation of receptorregulated SMAD transcription factors (R-SMADs) at their conserved C-terminal SXS motif (tail phosphorylation) by the type I receptors ${ }^{5}$. TGF $\beta$ ligands are divided into two subfamilies. The TGF $\beta$ subfamily primarily signals through the phosphorylation of SMADs 2 and 3, whereas the bone morphogenetic protein (BMP) subfamily signals through SMADs 1/5/8 (ref. 5). Once phosphorylated, R-SMADs interact with SMAD4 and translocate to the nucleus, where together with other cofactors, they regulate the transcription of over 500 target genes ${ }^{5-7}$.

Complex mechanisms, which are often context dependent, have evolved to check and modulate the potent activity of TGF $\beta$ ligands in controlling cell behaviour and tissue homoeostasis ${ }^{8,9}$. Although the regulation of the TGF $\beta$ pathway occurs at multiple layers of the signalling cascade, R-SMADs, as critical mediators of TGF $\beta$ signals, are suitably primed for key regulatory inputs. Indeed, reversible phosphorylation and ubiquitylation of SMAD proteins are critical processes that regulate the potency and duration of TGF $\beta$ signalling ${ }^{10,11}$. The mechanisms of ubiquitin-mediated turnover of R-SMADs by distinct E3 ubiquitin ligases are generally well established ${ }^{10,12-16}$. In particular, phosphorylation of the linker region of R-SMADs by CDK8/9, mitogen-activated protein kinases and GSK-3 marks R-SMADs for recognition by E3 ubiquitin ligases SMURF1/NEDD4L, which mediate their polyubiquitylation and degradation ${ }^{14,16,17}$. On the other hand, the turnover of active SMADs is likely to be regulated either by removal of the polyubiquitin chains by selective deubiquitylating enzymes (DUBs) or prevention of polyubiquitylation to produce a dynamic finetuning of signalling. In the TGF $\beta$ pathway, candidate proteins that recognize active SMADs and either deubiquitylate or prevent their ubiquitylation remain elusive.

A proteomic screen undertaken to identify novel interactors of R-SMADs isolated OTUB1 (OTU domain, ubiquitin aldehyde binding 1) in green fluorescent protein (GFP)-SMAD3 immunoprecipitates only from cells treated with TGF $\beta$. OTUB1 belongs to the ovarian tumour domain protease (OTU) family of DUBs that comprises 14 other members ${ }^{18}$. The canonical mode of action for OTUB1 to function is as a cysteine protease that hydrolyses the isopeptide bond between ubiquitin and the target or another ubiquitin molecule. The catalytic triad of OTUB1 is composed of $\mathrm{D} 88 / \mathrm{C} 91 / \mathrm{H} 265$ in the OTU domain, and a ubiquitin-binding motif is present in the $\mathrm{N}$ terminus ${ }^{19-22}$. OTUB1 has been reported to deubiquitylate TRAF3/6 (ref. 23), oestrogen receptor- $\alpha$ (ref. 24), p53 (ref. 25), c-IAP1 (ref. 26) and act as a specific receptor for ubiquitylated GRAIL ${ }^{27}$ and stabilize active $\mathrm{RhoA}^{28}$. Recently, several studies have described a noncanonical mode of OTUB1 action in which OTUB1 inhibits the ubiquitylation of target proteins by binding to and inhibiting the E2 ubiquitin-conjugating enzyme UBE2N (also known as UBC13) independent of its catalytic activity ${ }^{29-32}$.

In the present study, we identify OTUB1 as a novel component of the TGF $\beta$-induced active R-SMAD complex and demonstrate that it is essential for TGF $\beta$-induced gene transcription and cellular migration. We propose that the ability of OTUB1 to interact with SMAD2/3 and inhibit their polyubiquitylation, independent of catalytic activity, is necessary and sufficient for the regulation of TGF $\beta$ signalling. Further, our study uncovers a signal-induced phosphorylation-dependent recruitment of OTUB1 to its target in the TGF $\beta$ pathway.

\section{Results}

Identification of OTUB1 as an interactor of GFP-SMAD3. To uncover novel modes of regulation of the TGF $\beta$ pathway, we employed a proteomic approach to identify interactors of SMAD3, a key mediator of the TGF $\beta$ ligands. As a control, we used SMAD1, a mediator of the BMP signals. Human Embryonic Kidney 293 (HEK293) cells stably expressing N-terminally GFPtagged SMAD1 or SMAD3 under the control of a tetracycline inducible promoter were generated as described previously ${ }^{33}$. GFP immunoprecipitates (IPs) were resolved by SDS-polyacrylamide gel electrophoresis (PAGE) and the interacting proteins were excised, digested with trypsin and identified by mass spectrometry (Fig. 1a). As expected, BMP and TGF $\beta$ treatment resulted in the phosphorylation of GFP-SMAD1 and GFP-SMAD3, respectively (Supplementary Fig. S1a). Previously reported R-SMAD interactors, including SMAD4, LEMD3, TRIM33 and SKI were identified in GFP-SMAD1 and GFPSMAD3 IPs as indicated (Fig. 1a) ${ }^{34-39}$. OTUB1 was identified as a novel interactor of GFP-SMAD3 only when cells were treated with TGF $\beta$ (Fig. 1a). Four OTUB1 tryptic peptides were identified representing $8 \%$ sequence coverage (Fig. 1a). OTUB1 is ubiquitously expressed in mouse tissues (Supplementary Fig. S1b) and across many human cell lines, with relatively high expressions observed in breast cancer cells (Supplementary Fig. S1c).

OTUB1 interacts with the active SMAD2/3/4 complex. To verify the ligand inducible interaction between OTUB1 and SMAD3 at the endogenous level, OTUB1 was immunoprecipitated from human keratinocyte HaCaT cell extracts. OTUB1 was efficiently immunoprecipitated with the anti-OTUB1 antibody but not with pre-immune IgG (Fig. 1b). TGF $\beta$ induces formation of the active phospho-SMAD2/3-SMAD4 transcription complex $^{34,35}$. Tail (TP)- and linker (LP)-phosphorylated SMAD3, total SMAD3, SMAD2-TP and total SMAD2 were all detected in OTUB1 IPs from cells stimulated with TGF $\beta$ but not from BMP or unstimulated controls (Fig. 1b). SMAD4 was also detected in OTUB1 IPs only on TGF $\beta$ stimulation (Fig. 1b), indicating that OTUB1 binds to the active SMAD2/3/4 complex. Endogenous SMAD1-TP and SMAD4 were not detected in OTUB1 IPs from control or BMP-treated extracts (Fig. 1b), suggesting that OTUB1 selectively recognizes the TGF $\beta$-induced SMAD complexes. SMAD7, a transcriptional target of both BMP and TGF $\beta$ signalling, was also not detected in OTUB1 IPs (Fig. 1b). None of the SMADs were detected in pre-immune sheep IgG IPs, employed as control (Fig. 1b).

We also investigated the kinetics of OTUB1-SMAD2/3 interaction on TGF $\beta$ stimulation in $\mathrm{HaCaT}$ cells. The binding of endogenous OTUB1 to the active SMAD2/3/4 complex occurred within $30 \mathrm{~min}$ of TGF $\beta$ stimulation and persisted through $6 \mathrm{~h}$ of continuous stimulation, closely mirroring the levels of SMAD2/3 tail phosphorylation (Fig. 1c).

The TGF $\beta$-induced interaction between OTUB1 and SMAD2/3 was also confirmed in primary cells derived from mice (Fig. 1d). SMAD2-TP and total SMAD2 were detected in OTUB1 IPs from primary bone marrow-derived macrophages (BMDMs) and mouse embryonic fibroblasts (MEFs) only when cells were treated with TGF $\beta$ (Fig. 1d).

Phosphorylation of SMAD2/3 is necessary for OTUB1 binding. As shown in Fig. 1, OTUB1 selectively binds components of the active phospho-SMAD2/3-SMAD4 complex; however, it was not clear whether SMAD4 was required for this interaction. 
a

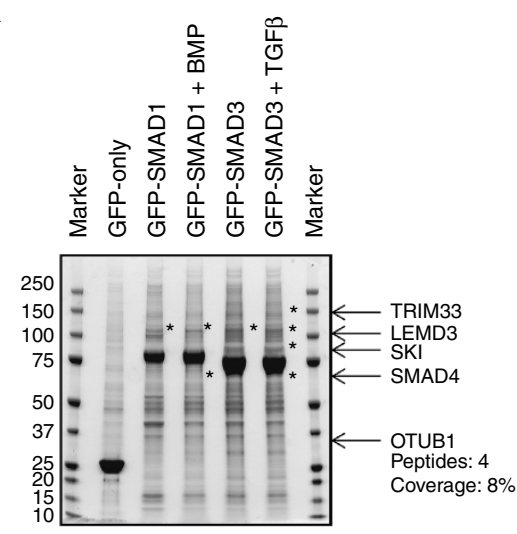

c

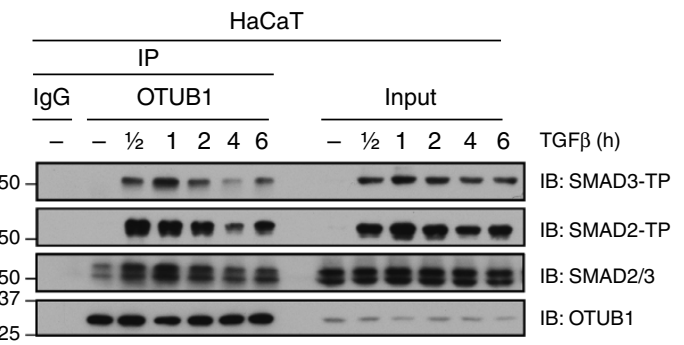

b

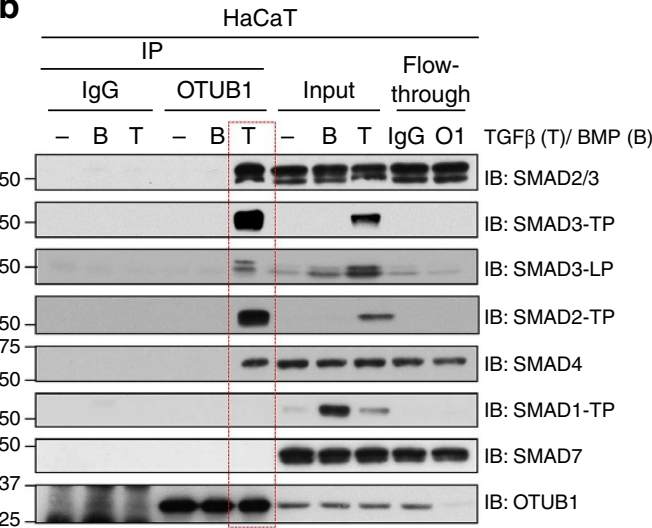

d

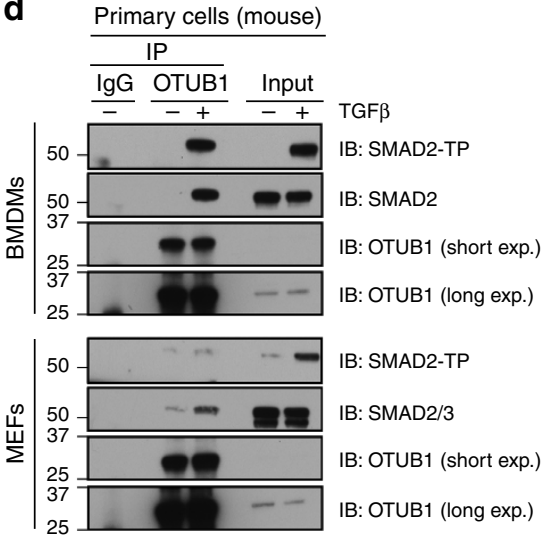

Figure 1 | OTUB1 interacts with TGF $\beta$-stimulated SMAD2/3/4. (a) HEK293 cells stably expressing GFP-only, GFP-SMAD1 or GFP-SMAD3 were treated with $50 \mathrm{pM} \mathrm{TGF} \beta$ or $25 \mathrm{ng} \mathrm{ml}^{-1}$ BMP for $1 \mathrm{~h}$ before lysis in the presence of dithiobis[succinimidyl propionate] (DSP). GFP immunoprecipitates (IPs) were separated by SDS-PAGE and interacting partners identified by mass spectrometry. (b) An endogenous IP with OTUB1 antibody or pre-immune sheep IgG was performed in $\mathrm{HaCaT}$ cell extracts, stimulated without or with $50 \mathrm{pM} \mathrm{TGF} \beta$ or $25 \mathrm{ng} \mathrm{ml}{ }^{-1} \mathrm{BMP}$ for $1 \mathrm{~h}$. Cell extracts (input), endogenous lgG or OTUB1 IPs and the corresponding immune-depleted flow-through extracts (O1=OTUB1-depleted untreated HaCaT extract) were resolved by SDS-PAGE and immunoblotted (IB) with the indicated antibodies. (c) As in $\mathbf{b}$, except that a time course of $50 \mathrm{pM}$ TGF $\beta$ treatment was performed for up to $6 \mathrm{~h}$ before lysis. (d) An endogenous IP with anti-OTUB1 antibody or pre-immune sheep IgG was performed in extracts from bone marrow-derived macrophages (BMDMs) and mouse embryonic fibroblasts (MEFs) stimulated with or without $50 \mathrm{pM}$ TGF $\beta$ for $1 \mathrm{~h}$. Before TGF $\beta$ stimulation, BMDMs were serum starved for $2 \mathrm{~h}$, whereas MEFs were not starved. Cell extracts (input) or IPs were resolved by SDS-PAGE and immunoblotted with the indicated antibodies. SMAD-LP, linker-phosphorylated SMAD; SMAD-TP, tail-phosphorylated SMAD.

Therefore, we employed the SW480 colon cancer cell line, which lacks SMAD4 expression ${ }^{40}$. In both $\mathrm{HaCaT}$ and SW480 cells, TGF $\beta$ induced the phosphorylation of SMAD2/3 (Fig. 2a). In both cells, phospho-SMAD2/3 proteins were detected in OTUB1 IPs on TGF $\beta$ stimulation (Fig. 2a). SW480 cells displayed elevated basal levels of phospho-SMAD2/3 over HaCaT cells (Fig. 2a). Consistent with this, even in the absence of TGF $\beta$ stimulation, phospho-SMAD2/3 were detected in OTUB1 IPs in SW480 cells (Fig. 2a). These observations imply that SMAD4 is not required for the interaction between OTUB1 and phospho-SMAD2/3.

To establish whether the phosphorylation of SMAD2/3 in response to TGF $\beta$ is critical for its interaction with OTUB1, we $\lambda$-phosphatase-treated OTUB1 IPs from TGF $\beta$-treated $\mathrm{HaCaT}$ extracts (Fig. 2b). This resulted in a significant dissociation of SMAD2 from OTUB1 IPs indicating that phosphorylation of SMAD2 in response to TGF $\beta$ is necessary for its interaction with OTUB1. As expected, $\lambda$-phosphatase efficiently dephosphorylated TGFß-induced phospho-SMAD2 in extracts (Fig. 2b; input). Further, endogenous OTUB1 IPs were capable of interacting with wild-type FLAG-SMAD3 and phospho-mimetic mutant of FLAG-SMAD3 but not phospho-deficient mutant of FLAGSMAD3 overexpressed in U2OS cells (Supplementary Fig. S1d). To definitively establish whether the interaction of OTUB1 with SMAD2 is tail phosphorylation dependent, we generated a biotin-tagged SMAD2 tail peptide (incorporating residues 428-467 of hSMAD2) phosphorylated at the $\mathrm{S}^{\star} \mathrm{MS}^{\star}$ motif (SMAD2 phospho-peptide). When the SMAD2 phospho-peptide was incubated with purified glutathione S-transferase-tagged (GSTOTUB1) protein in vitro, a robust OTUB1 interaction was observed (Fig. 2c; lane 2). This interaction was abolished upon $\lambda$-phosphatase treatment to dephosphorylate the SMAD2 phospho-peptide (Fig. 2c; lane 3). These observations suggest that the interaction between OTUB1 and phospho-SMAD2 is direct. Further, only SMAD2 phospho-peptide but not the $\lambda$-phosphatase-treated phospho-peptide was able to pull down endogenous OTUB1 from $\mathrm{HaCaT}$ cell extracts independent of TGF $\beta$ treatment (Fig. 2d). As expected, SMAD4, which is known to interact with SMAD2-TP ${ }^{35}$, also interacted with SMAD2 phospho-peptide (Fig. 2d).

OTUB1 enhances TGF $\beta$-mediated gene transcription. The ligand inducible interaction between OTUB1 and phosphoSMAD2/3 indicates that OTUB1 could have a regulatory role in the TGF $\beta$ pathway. We hypothesized that depleting OTUB1 

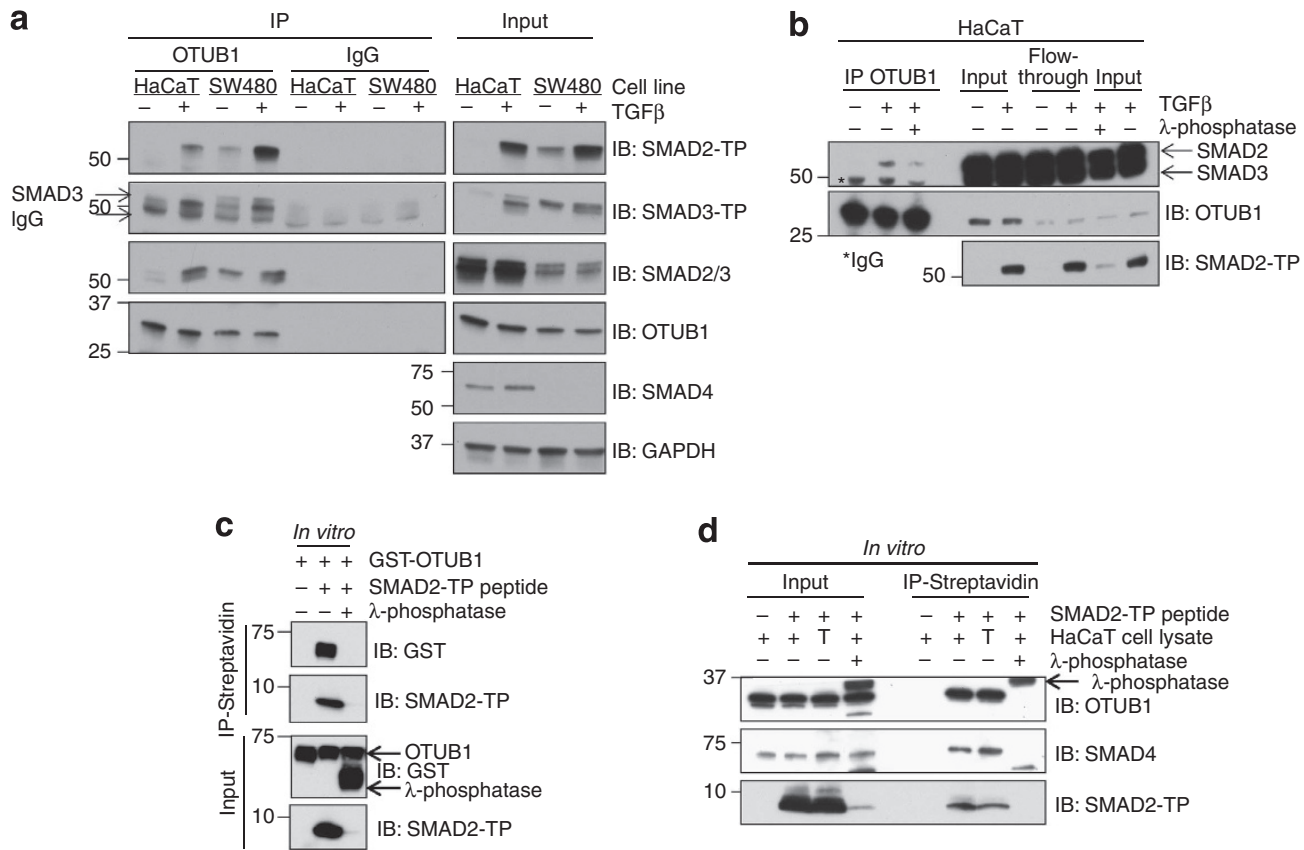

Figure 2 | OTUB1 binding to SMAD2/3 is phosphorylation dependent. (a) An endogenous IP with OTUB1 antibody or pre-immune sheep lgG was performed in $\mathrm{HaCaT}$ and SW480 cell extracts, stimulated with or without $50 \mathrm{pM}$ TGF $\beta$ for $1 \mathrm{~h}$ before lysis. Extracts (input) and endogenous OTUB1 or lgG IPs were resolved by SDS-PAGE and immunoblotted with the indicated antibodies. (b) Endogenous OTUB1 was immunoprecipitated from HaCaT cells treated with or without TGF $\beta(50 \mathrm{pM} ; 1 \mathrm{~h}$ ) before lysis. After washing, the IPs were then treated with or without lambda $(\lambda)$ phosphatase for 30 min at $30^{\circ} \mathrm{C}$ and washed four times. As a control, an aliquot of TGF $\beta$-treated cell extract was incubated with $\lambda$-phosphatase for 30 min at $30^{\circ} \mathrm{C}$. Cell extracts, IPs and flow-throughs were resolved by SDS-PAGE and immunoblotted with the indicated antibodies. (c) Purified GST-OTUB1 was incubated with biotintagged phospho-SMAD2 peptide in the presence or absence of $\lambda$-phosphatase for $30 \mathrm{~min}$ at $30^{\circ} \mathrm{C}$. The SMAD2 peptide was immunoprecipitated with streptavidin beads and washed six times. Input and IP samples were resolved by SDS-PAGE and immunoblotted with the indicated antibodies. (d) As in c, however, the SMAD2 peptide was incubated with untreated $\mathrm{HaCaT}(+)$ or TGF $\beta(\mathrm{T} ; 50 \mathrm{pM} ; 1 \mathrm{~h}$ )-treated cell lysate. SMAD4 antibody is used as a positive control.

expression from cells might promote the ubiquitylation of active phospho-SMAD2/3 and thus result in the inhibition of TGF $\beta$ signalling. Mouse myoblast $\mathrm{C} 2 \mathrm{C} 12$ cells stably integrated with a TGF $\beta$-responsive CAGA-luciferase reporter construct were transfected with multiple short interfering RNAs (siRNAs) targeting Otub1 (Fig. 3a). Depletion of Otub1 with iOTUB1\#1, yields $>90 \%$ knockdown of Otub1 protein levels (Fig. 3a) resulting in significant inhibition of TGF $\beta$-induced CAGA-luciferase reporter activity compared with control siRNA (mouse iFoxO4; Fig. 3b). Similar results were obtained with $i O T U B 1 \# 3$, which yielded $>80 \%$ depletion in Otub1 expression (Fig. $3 \mathrm{a}$ and Supplementary Fig. S2). These results suggest that Otub1 enhances TGF $\beta$ induced transcriptional responses. To confirm the repression of TGF $\beta$-mediated transcription on OTUB1 depletion, we tested the expression of endogenous TGF $\beta$ target genes by quantitative reverse transcription (qRT)-PCR in $\mathrm{HaCaT}$ cells (Fig. 3c). HaCaT cells were transiently transfected with a control siRNA (human iFoxO4), human OTUB1\#1 siRNA or a stable short hairpin RNA $(\operatorname{sh} R N A)$ against OTUB1. On depletion of OTUB1 by both transient and stable knockdown methods, TGF $\beta$-induced PAI-1 messenger RNA expression was significantly inhibited (Fig. 3d). The expression of another TGF $\beta$ target gene, CTGF, was also significantly inhibited upon $s i R N A$-mediated depletion of OTUB1 (Fig. 3e).

These results demonstrate that depletion of OTUB1 by two independent RNAi (RNA interference) oligonucleotides in mouse and human cells causes inhibition of TGF $\beta$-induced transcriptional activity. To rule out the possibility that these effects were due to off-target effects of siRNAs, we performed rescue experiments by employing OTUB1 constructs harbouring siRNAresistant silent mutations. Reintroduction of siRNA-resistant full-length OTUB1 rescued the OTUB1 transcripts to $\sim 70 \%$ of the endogenous levels (Fig. 5d). Under these conditions, the inhibition of TGF $\beta$-induced PAI- 1 mRNA expression caused by OTUB1 depletion was significantly reversed (Fig. 5d).

OTUB1 activity and its impact on SMAD3 ubiquitylation. To investigate the molecular mechanisms by which OTUB1 regulates SMAD2/3 function, we generated several OTUB1 mutants. Mutants were selected to either abolish OTUB1 catalytic activity or disrupt the interaction of OTUB1 with phospho-SMAD2/3. The catalytic cysteine of OTUB1, C91, is present in the OTU domain of OTUB1 and forms a catalytic triad with D88 and $\mathrm{H} 265$, which come into close contact on protein folding ${ }^{21}$. These three residues were targeted for mutagenesis to potentially disrupt the catalytic activity of OTUB1. The $\mathrm{N}$ terminus of OTUB1 harbours a ubiquitin-binding domain, which is deleted in the OTUB1 $\Delta \mathrm{N}$ mutant ${ }^{19,30}$. We also generated several other point mutants of OTUB1 by random mutagenesis; however, only K71R was selected for this study for its suitable properties as discussed below. The relative locations of the key residues mutated for this study are indicated in a schematic representation of OTUB1 (Fig. 4a, Supplementary Table S1).

We assessed the catalytic activity of OTUB1 mutants described above by employing in vitro deubiquitylation assays on di- or polyubiquitin chains. Wild-type OTUB1 cleaved K48-linked diubiquitin (Fig. 4b) as well as longer K48-linked polyubiquitin chains (Supplementary Fig. S3a), but not K63-linked polyubiquitin chains (Supplementary Fig. S3b). OTUB1 $\Delta \mathrm{N}$ mutant exhibited slightly reduced catalytic activity against K48-linked ubiquitin chains (Fig. 4b and Supplementary Fig. S3a), whereas all 
a

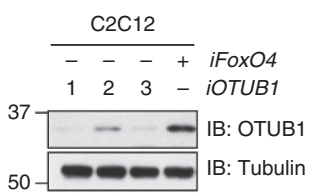

b

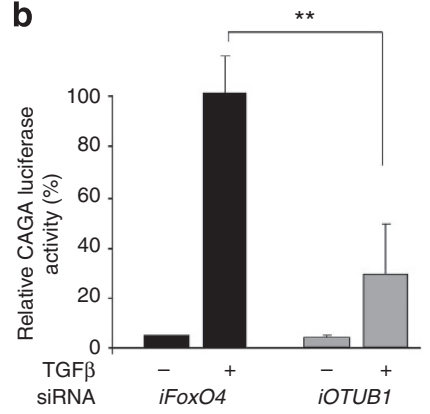

C

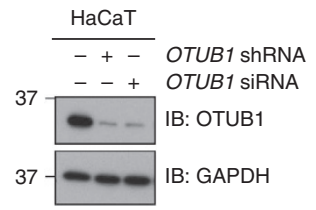

d
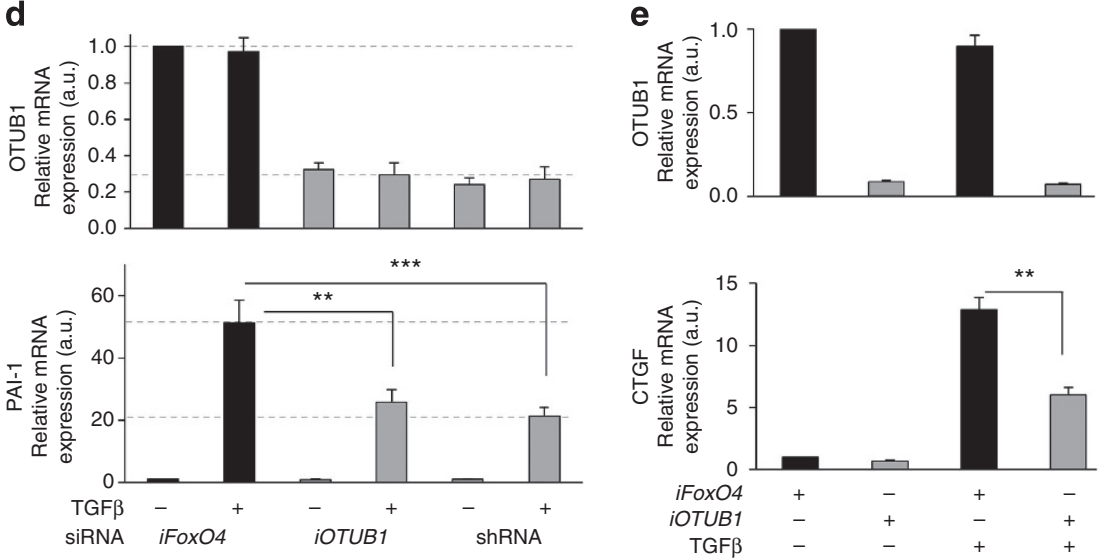

Figure 3 | Depletion of OTUB1 represses TGF $\beta$-induced transcription. (a) C2C12 cells were transfected with three different siRNAs (\#1, \#2 and \#3) targeting mouse OTUB1 (300 pM/10-cm dish each) and lysed $48 \mathrm{~h}$ after transfection. Extracts were resolved by SDS-PAGE and immunoblotted with OTUB1 and tubulin antibodies. (b) C2C12 cells stably expressing a SMAD3-dependent TGF $\beta$-responsive CAGA-luciferase reporter construct were transfected with iFoxO4 or iOTUB1\#1. Cells were treated with or without $50 \mathrm{pM}$ TGF $\beta$ for $6 \mathrm{~h}$ before lysis and luciferase activity was measured. Data are represented as mean and error bars indicate s.d. $(n=3)$. (c) HaCaT cells stably expressing shRNA against OTUB1 or transfected with control $(-)$ or OTUB1 siRNA (300 pM/10-cm dish each) for $48 \mathrm{~h}$ were lysed, and extracts were resolved by SDS-PAGE and immunoblotted with OTUB1 and GAPDH antibodies. (d) $\mathrm{HaCaT}$ cells, transfected with human OTUB1 siRNA, human FoxO4 siRNA, or stably expressing OTUB1 shRNA, were treated with $50 \mathrm{pM}$ TGF $\beta$ for $4 \mathrm{~h}$ before RNA isolation. Relative expression levels of indicated mRNAs were analysed by qRT-PCR. Data are represented as mean and error bars indicate s.d. $(n=6)$. (e) HaCaT cells depleted of human OTUB1 or FoxO4 by RNAi were treated with 50 pM TGF $\beta$ for 1 h. TGF $\beta$ was then washed off and SB505124 $(1 \mu \mathrm{M})$ added. RNA was isolated 45 min after TGF $\beta$ removal. Relative expression levels of OTUB1 and CTGF mRNAs were analysed by qRT-PCR. Data are represented as mean and error bars indicate s.d. $(n=6)$. Statistical significance of differences between experimental groups was assessed with Student's t-test. ${ }^{\star \star} P<0.01$ and ${ }^{\star \star \star} P<0.001$.

the other mutants of OTUB1, D88A, C91S, H265A, D88A/ H265A (D/H), D88A/C91S/H265A (D/C/H; Fig. 4b) and K71R (Fig. 4c) did not cleave K48-linked diubiquitin chains, indicating that these mutants were catalytically inactive. We also tested their ability to interact with and deubiquitylate SMAD3 in HEK293 cells. To test this, HEK293 cells were transfected with haemagglutinin (HA)-OTUB1 or mutant constructs together with FLAG-SMAD3 and HA ubiquitin. Full-length HA-OTUB1, as well as HA-OTUB1 $\Delta \mathrm{N}$ interacted with FLAG-SMAD3, whereas HA-OTUB1 C91S and HA-OTUB1 D/C/H mutants did not (Fig. 4d). Co-expression of HA-OTUB1 and FLAG-SMAD3 in HEK293 cells resulted in a spontaneous tail phosphorylation of FLAG-SMAD3 even in the absence of TGF $\beta$ (Supplementary Fig. S3c). This potentially explains the interaction between HA-OTUB1 and FLAG-SMAD3 under overexpression conditions even in the absence of TGF $\beta$ (Fig. $4 \mathrm{~d}$ and Supplementary Fig. S3c). HA-OTUB1 H265A and HA-OTUB1 K71R mutants also interacted with FLAG-SMAD3 (Fig. 4f). The interaction of HA-OTUB1 and mutants with FLAG-SMAD3 is not influenced in the absence or presence of transfected HA-ubiquitin (Supplementary Fig. S4a).

When co-expressed with HA-ubiquitin in HEK293 cells, efficient polyubiquitylation was observed in FLAG-SMAD3 IPs independent of TGF $\beta$ stimulation (Fig. 4d,f). On co-expression with wild-type OTUB1, a significant reduction in polyubiquitylation of FLAG-SMAD3 IPs was observed (Fig. 4d,e). However, this reduction was most probably because of the general reduction in polyubiquitin chains observed in extracts when OTUB1, or catalytically inactive mutants of OTUB1 (C91S and H265A), were overexpressed (Fig. 4d,e). Overexpression of the OTUB1 K71R mutant, which is also catalytically inactive but still interacts with SMAD3, only caused a moderate decrease in FLAG-SMAD3polyubiquitylation levels (Fig. 4e). Overexpression of OTUB1 also led to a general reduction in global endogenous polyubiquitylation in extracts (Supplementary Fig. S4a,b). This loss was not rescued completely by proteasomal inhibitors bortezomib and lactacystin (Supplementary Fig. S4a,b).

OTUB1 prevents SMAD3 ubiquitylation. The general loss of overall polyubiquitylation in whole-cell extracts on OTUB1 overexpression led us to test whether OTUB1 was capable of deubiquitylating polyubiquitylated SMAD2/3. FLAG-SMAD2/3 and SMAD4 overexpressed in HEK293 cells together with HAubiquitin and HA-NEDD4L (E3) were immunoprecipitated and used as a substrate for an OTUB1 in vitro deubiquitylation assay. Before lysis, cells were treated with TGF $\beta$ and bortezomib to induce SMAD2/3/4 complex formation and inhibit proteasomal 
a

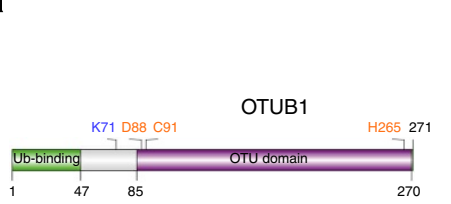

d

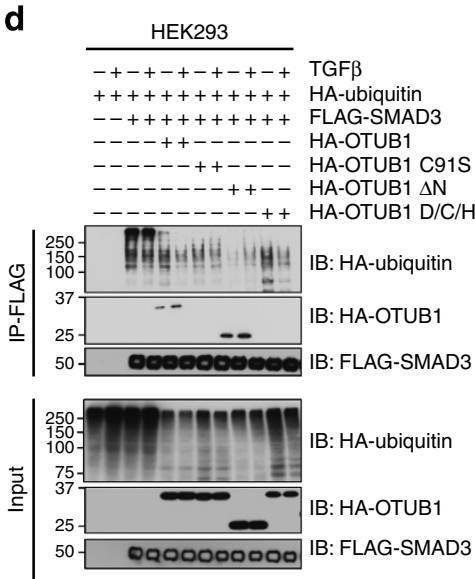

b

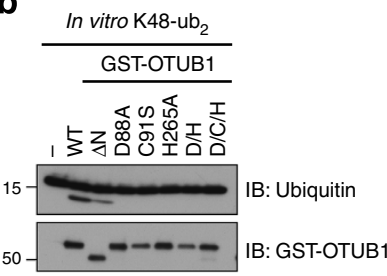

e
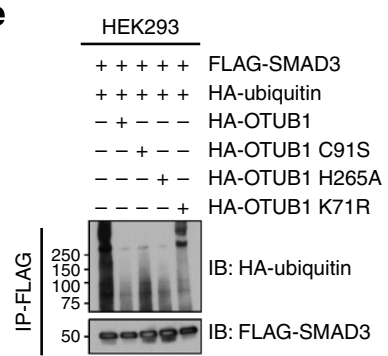

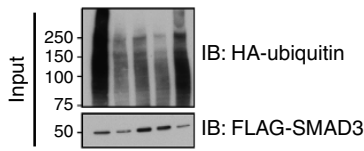

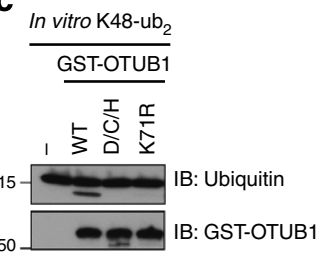

f

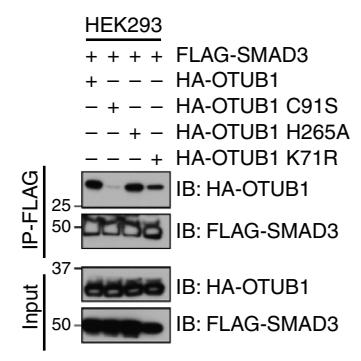

Figure 4 | Characterization of OTUB1 activity in vitro (a) A schematic representation of OTUB1 indicating the positions of key residues and domains. $(\mathbf{b}, \mathbf{c})$ Human recombinant GST-OTUB1 or indicated GST-OTUB1 mutants were incubated with K48-linked di-ubiquitin chains in a DUB assay buffer for $1 \mathrm{~h}$ at $30^{\circ} \mathrm{C}$. The reaction was stopped by the addition of $1 \times$ SDS sample buffer and the assay mix was resolved by SDS-PAGE and immunoblotted with GST or ubiquitin antibodies as indicated. (d-f) HEK293 cells were co-transfected with vectors encoding N-terminal HA-tagged OTUB1 or indicated HA-OTUB1 mutants (C91S, $\triangle \mathrm{N}$, D88A/C91S/H265A (D/C/H), H265A, K71R) and N-terminal FLAG-tagged SMAD3. Before lysis (in the presence of iodoacetamide), cells were treated with or without $50 \mathrm{pM}$ TGF $\beta$ and $10 \mu \mathrm{M}$ bortezomib for $3 \mathrm{~h}$. FLAG immunoprecipitates (IP) or extracts were resolved by SDS-PAGE and immunoblotted with the indicated antibodies.

degradation, preserving K48-linked polyubiquitin chains, respectively (Fig. 5a) ${ }^{41}$. On co-expression in HEK293 cells, NEDD4L induces K48-linked polyubiquitin chains on FLAGSMAD3 (Supplementary Fig. S5a), and E3-mediated degradation of endogenous SMAD2/3 can be rescued by bortezomib (Supplementary Fig. S5b). Wild-type OTUB1 and the $\Delta \mathrm{N}$ mutant, both capable of cleaving K48-linked di- or polyubiquitin chains in vitro, along with all the catalytically inactive mutants (D88A, C91S, H265A, D/H, D/C/H, K71R) were not able to deubiquitylate the polyubiquitylated FLAG-SMAD2/3/4 complex (Fig. 5a). Identical results were obtained when in vitro-polyubiquitylated recombinant SMAD3 was used as a substrate for OTUB1 (Supplementary Fig. S5c), indicating that OTUB1 does not deubiquitylate polyubiquitylated SMAD3.

This led us to hypothesize that OTUB1 might inhibit the ubiquitylation of SMAD2/3. The ability of OTUB1 to inhibit the ubiquitylation of target proteins independent of its catalytic activity has been reported before ${ }^{30}$. We polyubiquitylated recombinant SMAD2 in vitro using UBE1 (E1), UBE2D1 (E2) and NEDD4L (E3) (refs 14,42) (Fig. 5b). OTUB1 or OTUB1 C91S were added at increasing doses at the start of the reaction. In the absence of SMAD2, no autoubiquitylation of E2 or E3 is detected (Fig. 5b; lane 1). In the presence of SMAD2, robust polyubiquitylation is observed (Fig. 5b; lane 2). Adding increasing amounts of wild-type OTUB1 or catalytically inactive OTUB1 C91S at the start of the assay inhibits polyubiquitylation of SMAD2 in a dose-dependent manner (Fig. 5b; lanes 3-10). We also tested the ability of OTUB1 and mutants to inhibit the polyubiquitylation of FLAG-SMAD2/3/4 in vitro. As seen with recombinant SMAD2, TGF $\beta$-treated FLAG-SMAD2/3/4 IPs were polyubiquitylated in the presence of E1, E2 and E3. Polyubiquitylation was significantly inhibited when OTUB1 and most of the OTUB1 mutants (D88A, C91S, H265A, D/H) were added at the start of the ubiquitylation assay (Fig. 5c; lanes 4, 6-9). However, OTUB1 $\Delta \mathrm{N}, \mathrm{D} / \mathrm{C} / \mathrm{H}$ and $\mathrm{K} 71 \mathrm{R}$ were unable to inhibit polyubiquitylation (Fig. 5c; lanes 5, 10 and 11). These results imply that OTUB1 inhibits the ubiquitylation of SMAD2/3 rather than directly deubiquitylating them.

Consequently, we investigated how OTUB1 has an impact on the TGF $\beta$-induced transcription. We tested whether the catalytic activity of OTUB1, its ability to inhibit ubiquitylation of SMAD2/3 or its ability to interact with SMAD3 are essential to enhance TGF $\beta$-induced transcription. As described previously, the loss in expression of the TGF $\beta$ target gene PAI-1 caused by siRNAmediated depletion of OTUB1 is rescued by reintroduction of siRNA-resistant OTUB1 (Fig. 5d, Supplementary Fig. S5d). However, neither OTUB1 C91S (catalytically inactive, inhibits polyubiquitylation of SMAD3 in vitro but does not interact with SMAD3) nor OTUB1 $\Delta \mathrm{N}$ (catalytically active and interacts with SMAD3 but does not inhibit polyubiquitylation of SMAD3 in vitro) were able to rescue PAI-1 expression (Fig. 5d). This suggests that the ability of OTUB1 to both interact with and prevent the ubiquitylation of SMAD3 independent of its catalytic activity are essential for the regulation of TGF $\beta$-induced transcription.

OTUB1 prevents transfer of ubiquitin from E2s to E3s. OTUB1 has previously been reported to inhibit ubiquitylation of target proteins by interacting with and inhibiting E2 ubiquitin-conjugating enzymes ${ }^{29-32}$. Consistent with the reported findings ${ }^{30}$, we were able to identify E2 ubiquitin-conjugating enzymes, including UBE2N and all members of UBE2D and UBE2E family 
a

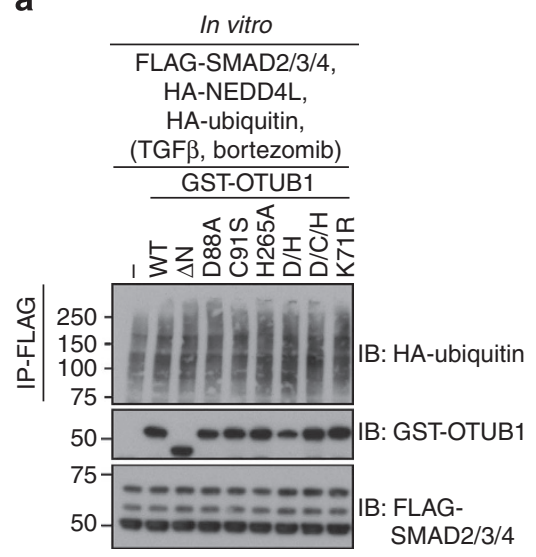

b

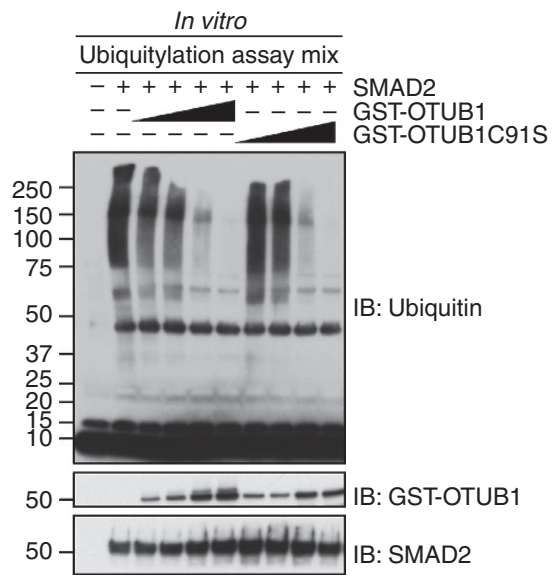

c

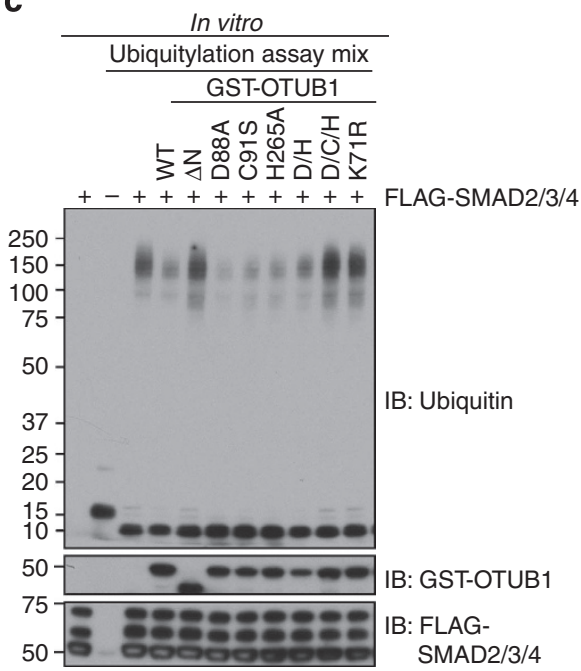

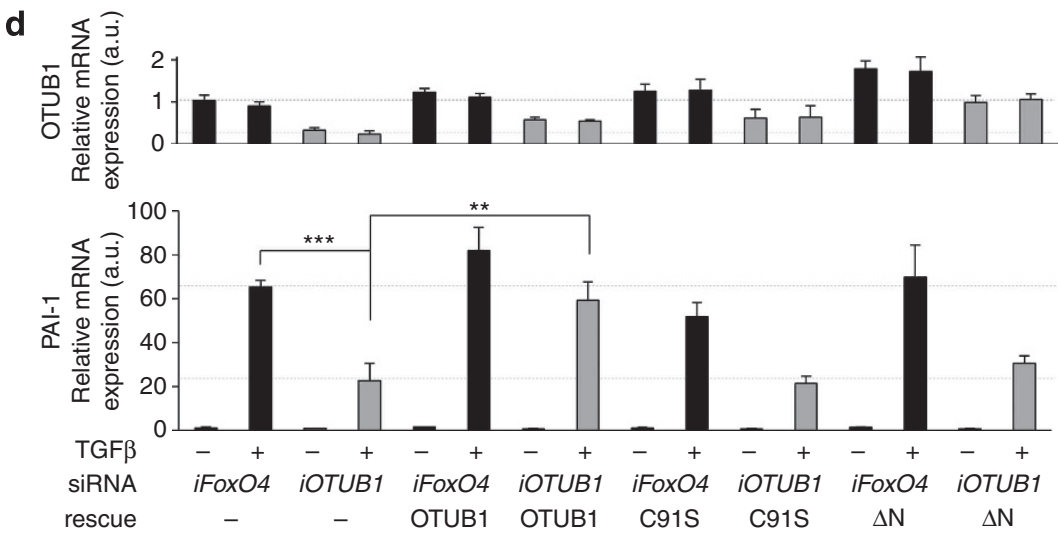

Figure 5 | OTUB1 prevents SMAD3 ubiquitylation in vitro. (a) For in-cell polyubiquitylation of FLAG-SMAD2/3/4, vectors encoding FLAG-SMAD2/3/4 were co-transfected with HA-NEDD4L and HA-ubiquitin in HEK293 cells and treated with $50 \mathrm{pM}$ TGF $\beta$ and $10 \mu \mathrm{M}$ bortezomib for $3 \mathrm{~h}$ before lysis and FLAG-SMAD2/3/4 were immunoprecipitated. An in vitro DUB assay of in-cell polyubiquitylated FLAG-SMAD2/3/4 was performed with GST-OTUB1 and the indicated GST-OTUB1 mutants in DUB assay buffer for $1 \mathrm{~h}$ at $30^{\circ} \mathrm{C}$. The assay mix was resolved by SDS-PAGE and immunoblotted with the indicated antibodies. (b) An in vitro ubiquitylation assay was performed with human recombinant SMAD2. SMAD2 was incubated with E1, E2, E3 and ubiquitin in ubiquitylation assay buffer for $1 \mathrm{~h}$ at $30^{\circ} \mathrm{C}$. Increasing concentrations of GST-OTUB1 and GST-OTUB1 C91S (8-60 ng $\mu \mathrm{I}^{-1}$ ) were added at the start of the ubiquitylation assay (time 0). Proteins were resolved by SDS-PAGE and immunoblotted with the indicated antibodies. (c) FLAG-SMAD2/3/4 IPs (from HEK293 cells expressing FLAG-SMAD2/3/4 treated with $50 \mathrm{pM}$ TGF $\beta$ for $1 \mathrm{~h}$ before lysis) were ubiquitylated in vitro in ubiquitylation assay buffer for $1 \mathrm{~h}$ at $30^{\circ} \mathrm{C}$ using E1, E2, E3 and ubiquitin. The indicated DUBs were added at the start of the ubiquitylation assay (time 0 ) and proteins were resolved by SDS-PAGE and immunoblotted with the indicated antibodies. (d) HaCaT cells were stably transfected with vectors encoding siRNA-resistant silent mutations (rescue) of the indicated OTUB1 constructs. These cells were then transfected with control FoxO4 or OTUB1 siRNA for $48 \mathrm{~h}$ and treated with or without TGF $\beta$ for $4 \mathrm{~h}$ before RNA isolation. Relative expression levels of indicated mRNAs were analysed by qRT-PCR. Data are represented as mean and error bars indicate s.d. $(n=6)$. Statistical significance of differences between experimental groups was assessed with Student's $t$-test. ${ }^{\star \star} P<0.01$ and ${ }^{\star \star \star} P<0.001$.

of E2s, as major interactors of GFP-OTUB1 by mass spectrometry (Supplementary Fig. S6 and Supplementary Table S2). In addition, we identified UBE2V1/2 and UBE2L3 as novel interactors of OTUB1 (Supplementary Table S2). Further, in endogenous OTUB1 IPs we were able to detect endogenous UBE2D and UBE2N independent of TGF $\beta$ signalling (Fig. 6a). To investigate whether the inhibition of E2 by OTUB1 was the rate-limiting step in its inhibition of SMAD3 polyubiquitylation in vitro, we set up an in vitro ubiquitylation assay with ubiquitin, E1, varying concentrations of E2, E3 and recombinant human SMAD3 in the presence or absence of $0.5 \mu \mathrm{M}$ GST-OTUB1 (Fig. 6b). In the absence of OTUB1, increasing concentrations of E2 resulted in enhanced polyubiquitylation of SMAD3 in a dose-dependent manner (Fig. 6b). As observed previously (Fig. 5b,c), the presence of OTUB1 in the reaction resulted in a significant inhibition of polyubiquitylation especially at lower concentrations of E2
(Fig. 6b). Higher concentrations of E2 substantially rescued this inhibition suggesting that binding of OTUB1 to E2 is likely to be the rate-limiting step in the inhibition of polyubiquitylation of SMAD3 (Fig. 6b). We next investigated whether OTUB1 inhibits E2 by preventing the conjugation of ubiquitin or the transfer of ubiquitin from E2 to E3. In the presence of ubiquitin, ATP and E1, almost every molecule of E2 becomes loaded with ubiquitin (Fig. 6c; compare lane 1 with 2). This ubiquitin loading of E2 is not inhibited when wild-type OTUB1, OTUB1 C91S or OTUB1 K71R mutants are present. When E3 ligase NEDD4L and its substrate SMAD3 are added to the reaction, in the absence of OTUB1 most of the ubiquitin at the predicted molecular weight disappears (Fig. 6c; compare lane 2 with 7). Under these conditions, consistent with an efficient transfer of ubiquitin from E2-Ub to E3 and substrate, the E2 is mostly observed in a non-ubiquitin-loaded native molecular weight state (Fig. 6c). 


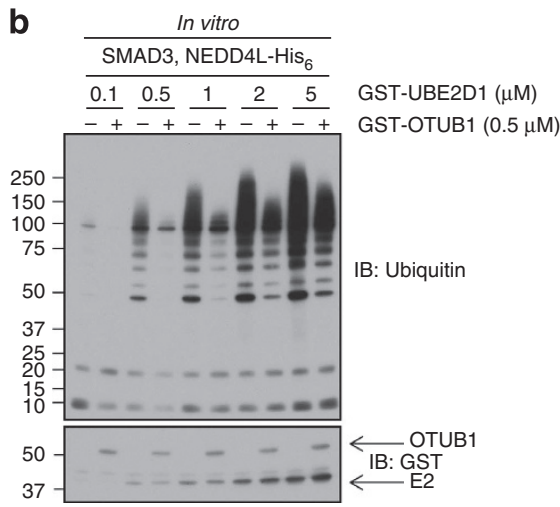

C

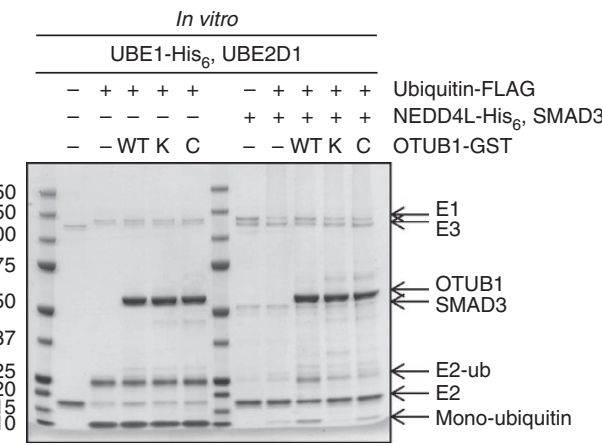

Figure 6 | OTUB1 interacts with E2 enzymes and inhibits efficient ubiquitin transfer from E2 to E3. (a) An endogenous IP with OTUB1 antibody or pre-immune sheep IgG was performed in HaCaT cell extracts stimulated with or without $50 \mathrm{pM}$ TGF $\beta$ for $1 \mathrm{~h}$ before lysis in the presence of dithiobis[succinimidyl propionate] (DSP). Cell extracts (input), endogenous IgG or OTUB1 IPs and the corresponding immune-depleted flow-through extracts were resolved by SDS-PAGE and immunoblotted with the indicated antibodies. (b) An in vitro ubiquitylation assay was performed with human recombinant SMAD3, His-E1 $(0.1 \mu \mathrm{M})$, ubiquitin, His-E3 $(1 \mu \mathrm{M})$ and increasing concentrations of GST-E2 $(0.1-5 \mu \mathrm{M})$ in ubiquitylation assay buffer for $1 \mathrm{~h}$ at $30^{\circ} \mathrm{C}$. GST-OTUB1 was added at the start of the ubiquitylation assay (time 0). Proteins were resolved by SDS-PAGE and immunoblotted with the indicated antibodies. (c) His-E1, E2, FLAG-ubiquitin, GST-OTUB1 and mutants were mixed with or without His-E3 and SMAD3 before the addition of ATP. After $10 \mathrm{~min}$ at $30^{\circ} \mathrm{C}$, proteins were separated by SDS-PAGE and visualized with Coomassie Instantblue.

However, when wild-type OTUB1 is added to the reaction at the start, some ubiquitin is observed at its native molecular weight, whereas a significant amount of ubiquitin-loaded E2 is also observed, suggesting that OTUB1 inhibits the transfer of ubiquitin from the E2-Ub complex to E3 (Fig. 6c).

OTUB1 C91S, a catalytically inactive mutant that does not interact with SMAD3 but prevents its ubiquitylation, mimics wild-type OTUB1. However, OTUB1 K71R, a catalytically inactive mutant, does not appear to inhibit the transfer of ubiquitin from the E2-Ub complex to E3 (Fig. 6c cf. bottom line mono-ubiquitin). This is consistent with the inability of OTUB1 K71R mutant to inhibit the ubiquitylation of SMAD3 (Fig. 5c). These results suggest that by binding to E2 ubiquitin-conjugating enzymes, OTUB1 appears to inhibit the transfer of ubiquitin from E2-Ub complex onto E3 ubiquitin ligases.

OTUB1 rescues phospho-SMAD2/3 from proteasomal degradation. We have demonstrated that OTUB1 binds to phospho-SMAD2/3 and E2 ubiquitin-conjugating enzymes, thereby inhibiting the polyubiquitylation of $\mathrm{SMAD} 2 / 3$ in vitro. Therefore, we reasoned that OTUB1 enhances the TGF $\beta$-induced transcriptional responses by stabilization of the active phosphoSMAD2/3 pool in cells by protecting it from polyubiquitinmediated proteasomal degradation.

To demonstrate that the turnover of active phospho-SMAD2/3 is mediated in part by proteasomal degradation, $\mathrm{HaCaT}$ cells were treated with $26 \mathrm{~S}$ proteasome inhibitors (bortezomib or MG132) and TGF $\beta$ for $3 \mathrm{~h}$. Bortezomib, and to a lesser extent MG132, treatment resulted in enhanced levels of TGF $\beta$-induced phosphoSMAD2/3 as well as polyubiquitylated proteins (Supplementary Fig. S7a). However, when TGF $\beta$ and bortezomib were added to the cells for $1 \mathrm{~h}$, the increase in the levels of TGF $\beta$-induced phosphoSMAD2/3 caused by bortezomib was only slight. In addition, early induction of phospho-SMAD2/3 by TGF $\beta$ was unaffected by siRNA-mediated depletion of OTUB1 (Fig. 7a). As polyubiquitylation and degradation of phospho-SMAD2/3 occurs subsequent to the assembly of the active SMAD2/3/4 complex at the transcription sites ${ }^{14}$, we performed a pulse-chase experiment. Control or OTUB1-depleted HaCaT cells were stimulated with TGF $\beta$ for $1 \mathrm{~h}$ to induce maximal phosphorylation of SMAD2/3, after which the TGF $\beta$ ligand was removed. The levels of phospho-SMAD2/3 were tracked at fixed time points thereafter in the presence or absence of bortezomib. The cells depleted of OTUB1 expression exhibited lower levels of phospho-SMAD2/3 after $30 \mathrm{~min}$ and $1 \mathrm{~h}$ of TGF $\beta$ removal compared with control cells (Fig. 7b; lanes 2 and 6). This effect appeared to be a result of rapid proteasomal degradation, as the levels of phospho-SMAD2/3 are stabilized in the presence of the proteasomal inhibitor bortezomib (Fig. 7b; lanes 4 and 8). The levels of total SMAD2/3 or SMAD4 did not change significantly (Fig. 7b). Bortezomib stabilized phospho-SMAD2/3 levels for up to $16 \mathrm{~h}$ following ligand removal (Supplementary Fig. S7b). The reduction in the levels of phospho-SMAD2 caused by depletion of OTUB1 was rescued by a siRNA-resistant wild-type FLAG-OTUB1 construct, suggesting that the results obtained were unlikely to be due to the off-target effects (Fig. 7c).

TGF $\beta$ signalling has a critical role in multiple cellular processes, including wound healing and migration $3,43-45$. 
a

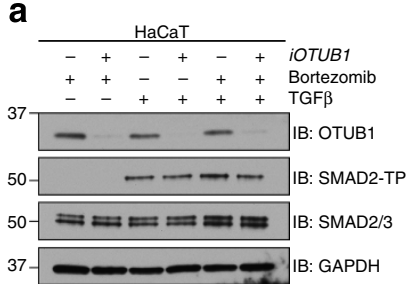

b

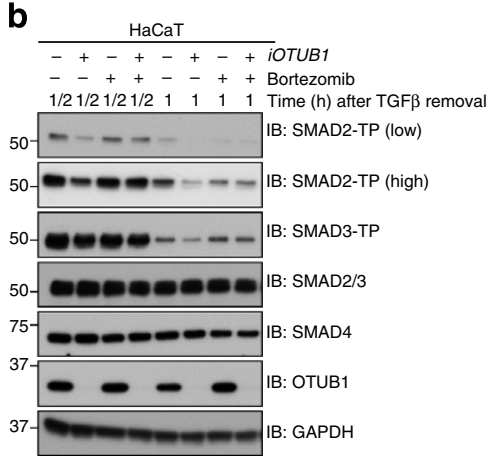

c
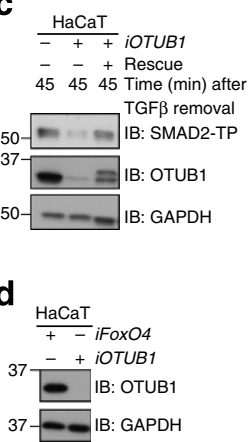
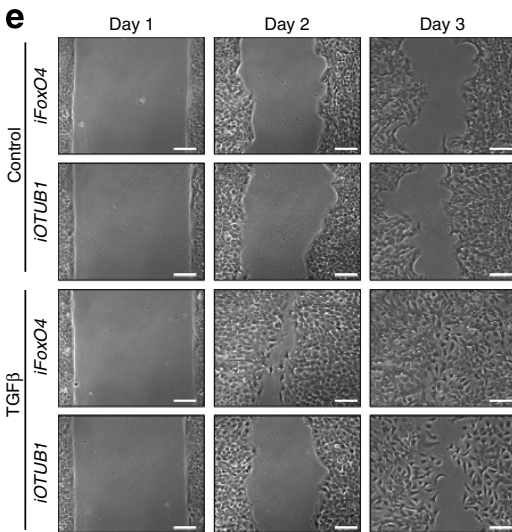

f
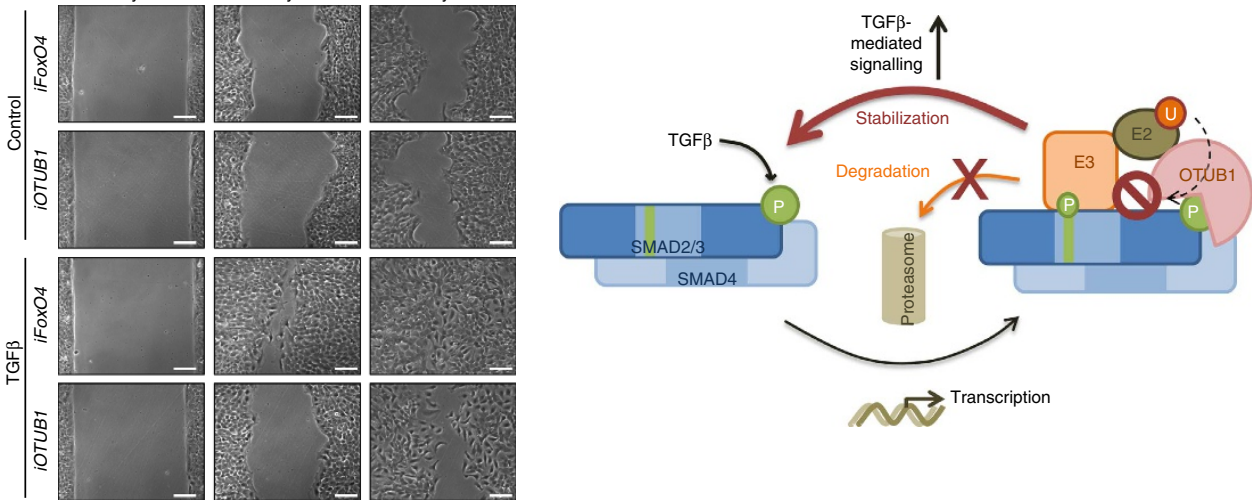

Figure 7 | OTUB1 protects phospho-SMAD2/3 from proteasomal degradation and has an impact on TGF $\beta$-induced cell migration. (a) HaCaT cells transfected with FoxO4 ( - ) or OTUB1 siRNAs were treated with TGF $\beta(50 \mathrm{pM})$ and bortezomib $(10 \mu \mathrm{M})$ for $1 \mathrm{~h}$. Extracts were resolved by SDS-PAGE and immunoblotted with the indicated antibodies. (b) HaCaT cells, expressing control shRNA ( - ) or OTUB1 shRNA were transfected with FoxO4 ( - ) or OTUB1 siRNAs, respectively, $48 \mathrm{~h}$ before cell lysis. Cells were serum starved for $16 \mathrm{~h}$ and stimulated with TGF $\beta$ ( $50 \mathrm{pM})$ and bortezomib (10 $\mu \mathrm{M})$ for $1 \mathrm{~h}$. The cells were then washed in PBS and media was replaced with starvation media supplemented with SB505124 ( $1 \mu$ M; to block any further TGF $\beta$ receptor activity), with or without bortezomib $(10 \mu \mathrm{M})$. The cells were lysed at $1 / 2$ or $1 \mathrm{~h}$ time points and cell extracts resolved by SDS-PAGE and immunoblotted with the indicated antibodies. (c) As in $\mathbf{b}$, except cells were transfected with vector encoding FLAG-tagged full-length OTUB1 resistant to OTUB1 shRNAs and siRNAs (rescue). (d) HaCaT cells used in e were lysed after $48 \mathrm{~h}$ and extracts were resolved by SDS-PAGE and immunoblotted with the indicated antibodies, confirming a knockdown of OTUB1. (e) HaCaT cells stably expressing control shRNA and FoxO4 siRNA or OTUB1 shRNA and OTUB1 siRNA were seeded onto migration inserts (Ibidi). After $24 \mathrm{~h}$, the inserts were removed and cells were serum starved for $4 \mathrm{~h}$ and stimulated without or with TGF $\beta$ $(50 \mathrm{pM})$. Cell migration was tracked by taking images of the gaps every $24 \mathrm{~h}$. Scale bars, $100 \mu \mathrm{m}$. (f) A schematic representation of OTUB1 function within the TGF $\beta$ signalling pathway. TGF $\beta$ induces SMAD2/3 tail phosphorylation, association with SMAD4 and entry into the nucleus, where SMADs act as transcription factors. Following linker phosphorylation, E3 ubiquitin ligase NEDD4L recognizes the SMAD2/3/4 complex and polyubiquitylates SMAD2/3, leading to proteasomal degradation. OTUB1 interacts with, and thereby chaperones, the active SMAD2/3 complex by inhibiting its ubiquitylation, through its interactions with and inhibition of cognate E2. Proteasomal degradation of active SMAD2/3 is thus prevented and the complex can recycle, which leads to sustained TGF $\beta$ signalling.

The induction of cellular migration by TGF $\beta$ is in part implicated in cancer progression and metastasis ${ }^{3,45}$. To assess the impact of OTUB1 on TGF $\beta$-induced cellular migration, a wound-healing 'scratch assay' was employed. Control or OTUB1-depleted $\mathrm{HaCaT}$ cells (Fig. 7d) were cultured to confluency in adjacent chambers of an insert separated by a small fixed-sized spacer. On removal of the insert, a uniform gap was formed. Cellular migration onto the gap was monitored for up to $48 \mathrm{~h}$ (Fig. 7e). In control cells, TGF $\beta$ treatment induced migration of cells within $24 \mathrm{~h}$, and by $48 \mathrm{~h}$ the gap was completely covered with cells. Depletion of OTUB1 substantially inhibited the migration of $\mathrm{HaCaT}$ cells onto the gap after TGF $\beta$ treatment (Fig. 7e).

\section{Discussion}

This study uncovers an intriguing regulatory role for OTUB1 in recognizing TGF $\beta$-induced phospho-SMAD2/3, controlling their turnover and consequently modulating pathway signalling. The results reveal for the first time a remarkable interplay between phosphorylation and the recruitment of OTUB1 to its target in the TGF $\beta$ pathway. The findings shed light into the molecular mechanisms by which the phospho-SMAD2/3-OTUB1 interaction results in the regulation of TGF $\beta$-induced transcription as well as phospho-SMAD2/3 turnover.

TGF $\beta$-induced phosphorylation of SMAD2/3 by the type I TGF $\beta$ receptor kinases is a fundamental feature in the intracellular transduction of TGF $\beta$ ligands. This induces the formation of an active SMAD2/3/4 transcription complex, translocation to the nucleus and transcriptional control of target genes ${ }^{5}$. OTUB1 was isolated from a proteomic screen as an interactor of GFPSMAD3 only when cells were treated with TGF $\beta$. Remarkably, endogenous OTUB1 interacts with SMAD2/3 only on TGF $\beta$ induction (Fig. 1b,d), and the levels of phospho-SMAD2/3 on TGF $\beta$ stimulation directly correlate with the ability of OTUB1 to pull down SMAD2/3 (Fig. 1c). SMAD4 was also detected in OTUB1 immunoprecipitates on TGF $\beta$ stimulation (Fig. 1b). However, even in the absence of SMAD4, OTUB1 interacts with TGF $\beta$-induced phospho-SMAD2/3, indicating that SMAD4 is 
not necessary to mediate phospho-SMAD2/3-OTUB1 interaction (Fig. 2a). Consistent with the notion that OTUB1 interaction could be phospho-SMAD2/3-dependent, dephosphorylation of OTUB1 IPs by $\lambda$-phosphatase to dephosphorylate phosphoSMAD2/3 partially abolishes OTUB1-SMAD2/3 interaction (Fig. 2b). Further, a SMAD2 tail phospho-peptide was able to interact with recombinant OTUB1 as well as endogenous OTUB1 from extracts indicating that tail phosphorylation of SMAD2 is sufficient for its interaction with OTUB1 (Fig. 2c,d).

Despite the phospho-dependent nature of OTUB1 interaction with SMAD2/3, no phospho-interaction motifs within OTUB1 have been reported. A phospho-SMAD3-OTUB1 structural analysis might shed light into the molecular basis of their interaction. To date, no phospho-dependent mode of OTUB1 recruitment to its target has been reported.

The TGF $\beta$-dependent recruitment of OTUB1, a DUB, combined with the inhibition of TGF $\beta$-induced transcriptional responses on OTUB1 depletion initially suggested that OTUB1 might have an impact on TGF $\beta$ signalling by deubiquitylating and stabilizing phosho-SMAD2/3 in cells. It is known that SMAD2/3-TP in the nucleus are targeted for polyubiquitylation by NEDD4L on further phosphorylation at the linker region by $\mathrm{CDK} 8 / 9$, which then leads to their proteasomal degradation (Supplementary Fig. S5a,b) ${ }^{14}$. In vitro OTUB1 cleaves K48-linked polyubiquitin chains, which in cells direct target proteins for proteasomal degradation ${ }^{46}$. However, OTUB1 was unable to deubiquitylate polyubiquitylated SMAD2/3 in vitro (Fig. 5a). Further investigations revealed that the action of OTUB1 predominantly relies on its ability to inhibit the ubiquitylation of SMAD2/3 in vitro rather than isopeptide cleavage (Fig. 5b,c). Further, some catalytically inactive mutants of OTUB1, including OTUB1 C91S, were also able to inhibit SMAD2/3 ubiquitylation in vitro (Fig. $5 \mathrm{c}$ ).

The ability of OTUB1 to inhibit the polyubiquitylation of chromatin on DNA damage independent of its catalytic activity has been shown previously ${ }^{30}$. This and other studies have reported that OTUB1 inhibits ubiquitylation by binding to and inhibiting E2 enzymes ${ }^{25,29-32}$. In agreement with these reports, we identified several E2 enzymes, including UBE2D1, as OTUB1-interacting partners from a proteomic screen. Moreover, we demonstrated the endogenous interactions between OTUB1 and UBE2N or UBE2D1 (Fig. 6a). We further showed that OTUB1, by binding to UBE2D1, inhibits the transfer of ubiquitin from E2-Ub conjugate to E3 ubiquitin ligase NEDD4L and subsequently to SMAD3 (Fig. 6c).

The activation of TGF $\beta$ signalling results in the transcription of target genes, which ultimately determines the nature of cellular responses. Our findings indicate that OTUB1 modulates TGF $\beta$ induced transcriptional responses. $R N A i$-mediated depletion of OTUB1 from $\mathrm{HaCaT}$ cells resulted in inhibition of TGF $\beta$ induced expression of PAI-1 and CTGF transcripts, both known TGF $\beta$ target genes (Fig. $3 \mathrm{~d}, \mathrm{e})^{47,48}$.

We were able to rescue the inhibition of TGF $\beta$-induced transcription on OTUB1 depletion by siRNA-resistant OTUB1 (Fig. 5d). However, neither siRNA-resistant OTUB1 C91S mutant (catalytically inactive, SMAD3-interaction deficient but able to inhibit SMAD3 ubiquitylation) nor OTUB1 $\triangle \mathrm{N}$ mutant (catalytically active, interacts with SMAD3 but unable inhibit SMAD3 ubiquitylation) was able to rescue TGF $\beta$-induced transcription caused by OTUB1 depletion (Fig. $5 \mathrm{~d}$ ). Together, these results imply that binding to SMAD2/3 as well as the ability to inhibit SMAD2/3 ubiquitylation are essential for OTUB1 to have an impact on the TGF $\beta$ pathway. Therefore, the association of OTUB1 with phospho-SMAD2/3 would be predicted to prevent SMAD $2 / 3$ from being polyubiquitylated and degraded, thereby enhancing TGF $\beta$ signalling. Consistent with this notion, the depletion of OTUB1 from cells leads to a rapid loss in levels of SMAD2/3-TP but the loss in levels are rescued on treatment of cells with proteasomal inhibitor bortezomib or expression of siRNA-resistant OTUB1 (Fig. 7b,c).

In this study, we have identified OTUB1 as a novel regulator of the TGF $\beta$ pathway. The TGF $\beta$ pathway controls multiple cellular processes and is implicated in carcinogenesis, in part through the induction of cellular migration ${ }^{3,45}$. We demonstrate that the depletion of OTUB1 from HaCaT cells significantly inhibits lateral migration induced by TGF $\beta$ (Fig. 7e). Our findings imply that OTUB1 enhances TGF $\beta$ signalling responses by interacting with and stabilizing SMAD2/3-TP, by preventing its ubiquitylation and degradation (Fig. 7f). These observations make OTUB1 a promising target for the development of small molecule inhibitors specifically targeting the OTUB1-SMAD2/3 interaction against diseases, such as cancer and fibrosis, where TGF $\beta$ signalling is abnormal.

\section{Methods}

Cell culture. All cells (HaCaT, HEK293, SW480, U2OS, C2C12) were cultured in DMEM (Gibco) supplemented with 10\% fetal bovine serum (Hyclone), $2 \mathrm{mM}$ L-Glutamine (Lonza) and $1 \%$ penicillin/streptomycin (Lonza) and maintained at $37^{\circ} \mathrm{C}$ in a humidified atmosphere with $5 \% \mathrm{CO}_{2}$. HEK293 cells were obtained from ATCC, HaCaT cells from J. Massague (New York), U2OS cells from J. Rouse (Dundee), SW480 cells from A. Prescott (Dundee) and C2C12 cells from G. Inman (Dundee). Primary cells (MEFs and BMDMs) were generated from animals that were housed under specific pathogen-free conditions in accordance with UK and EU regulations. Wild-type MEFs were isolated from decapitated embryos with red organs removed. The embryo was minced and resuspended in $1 \mathrm{ml}$ trypsin and incubated at $37^{\circ} \mathrm{C}$ for $15 \mathrm{~min}$ before the addition of $10 \mathrm{ml}$ growth medium. Cells were plated and allowed to attach overnight before cells were washed with fresh medium to remove debris. Growth media were additionally supplemented with $\mathrm{Na}$-pyruvate and non-essential amino acids. BMDMs were generated by flushing femurs from a female mouse with PBS. Cells were then pelleted by centrifugation and cultured on bacterial grade plastic plates for 7 days in media (additionally supplemented $0.25 \mathrm{\mu g} \mathrm{ml}^{-1}$ amphotericin and $5 \mathrm{ng} \mathrm{ml}^{-1}$ recombinant macrophage colony-stimulating factor (M-CSF)). Cells were then detached by scraping in versene (Invitrogen) and re-plated on tissue culture-treated petri dishes. All procedures were carried out in accordance with University of Dundee and United Kingdom Home Office regulations ${ }^{49,50}$. FlpIN TRex HEK293 cell lines (Invitrogen) stably expressing tetracycline inducible GFP constructs (GFP, GFP-SMAD1, GFPSMAD3 and GFP-OTUB1) were additionally grown in $100 \mu \mathrm{g} \mathrm{ml}^{-1}$ hygromycin B and $15 \mu \mathrm{g} \mathrm{ml}^{-1}$ blasticidin. GFP-protein expression was induced with $20 \mathrm{ng} \mathrm{ml}^{-1}$ doxycycline for $16 \mathrm{~h}$ before lysis. Stable cell lines infected with pBABE-puro (Cell Biolabs Inc.) retroviral constructs or pSUPERIOR-puro retroviral (Oligoengine) shRNA constructs were supplemented with $2 \mu \mathrm{g} \mathrm{ml}^{-1}$ puromycin. Stable CAGA (TGF $\beta$ responsive) luciferase reporter $\mathrm{C} 2 \mathrm{C} 12$ cells (obtained from Dr G. Inman) were selected in $0.7 \mathrm{mg} \mathrm{ml}^{-1} \mathrm{G} 418$ (Sigma). Cells were serum starved for $16 \mathrm{~h}$ at $37^{\circ} \mathrm{C}$ before ligand treatment with human recombinant $\mathrm{BMP}_{2}\left(25 \mathrm{ng} \mathrm{ml}^{-1}\right)$ or TGF $\beta_{1}$ (50 pM; R\&D Systems) for the indicated time points. SB505124 (Sigma), a specific TGF $\beta$-receptor ALK4/5/7 inhibitor, was used at $1 \mu \mathrm{M}$ for the indicated time points ${ }^{51}$. Proteasome inhibitors bortezomib (LC Laboratories), MG132 (Calbiochem) and lactacystin (Sigma) were used at $10 \mu \mathrm{M}$ for $3 \mathrm{~h}$.

Plasmid transfections were done using $25 \mu \mathrm{l}$ of $1 \mathrm{mg} \mathrm{ml}^{-1}$ polyethylenimine (Polysciences, in $25 \mathrm{mM}$ HEPES pH 7.5) in $1 \mathrm{ml}$ DMEM with a maximum of $10 \mu \mathrm{g}$ plasmid DNA ${ }^{52}$. All plasmids expressing mammalian proteins were cloned into pCMV5, pBABE-puro or pCDNA-Frt-TO vectors with N-terminal FLAG, GFP or HA tags. pCDNA-Frt-TO plasmids were used to generate stable tetracycline inducible HEK293 cell lines following the manufacturer's protocol (Invitrogen). pRetro SUPERIOR shRNA control or OTUB1 shRNA (5'-GCAAGTTCTTCGAG CACTT- $3^{\prime}$ ), as well as pBABE constructs (pBABE-puro control or OTUB1 or mutant constructs harbouring silent mutations for protection against human iOTUB1\#1 and/or \#3 siRNAs) were generated as retroviruses in HEK293T cells according to manufacturers' protocols and used to infect target cells ${ }^{53}$. Infected cells were selected and maintained in $2 \mu \mathrm{g} \mathrm{ml}^{-1}$ puromycin.

All DNA constructs used were verified by DNA sequencing, performed by DNA Sequencing \& Services (MRCPPU, College of Life Sciences, University of Dundee, Scotland, http://www.dnaseq.co.uk) using Applied Biosystems Big-Dye Ver 3.1 chemistry on an Applied Biosystems model 3730 automated capillary DNA sequencer.

For siRNA oligonucleotide transfections, the cells were transfected during attachment. Thirty microlitres of transfectin (Bio-Rad) and $300 \mathrm{pM}$ of siRNA (per $10-\mathrm{cm}$ diameter dish) were mixed in $2 \mathrm{ml}$ OptiMEM (Life Technologies). After incubating for $15 \mathrm{~min}$, the solution was added to the cells and cells were lysed $48 \mathrm{~h}$ after transfection. The following siRNA oligonucleotides (Sigma) were used: OTUB1 (\#1) : human: $5^{\prime}$-GCAAGUUCUUCGAGCACUU- $3^{\prime}$, mouse: $5^{\prime}$-GAACCC AUGUGCAAGGAGA-3', OTUB1 (\#3): human: 5'-CCGACUACCUUGUG GUCUA-3', mouse: $5^{\prime}$-CAAUUGAAGACUUCCACAA-3', FoxO4: human: $5^{\prime}$-CCCGACCAGAGAUCGCUAA-3', mouse: 5'-GCAAGUUCAUCAAGGU 
UCA-3'. FoxO4 siRNA was used as a control as it is known not to interfere with the BMP/TGF $\beta$ pathway ${ }^{33,54}$.

For lysis, cells were scraped on ice in lysis buffer $(50 \mathrm{mM}$ Tris- $\mathrm{HCl} \mathrm{pH} 7.5$, $0.27 \mathrm{M}$ sucrose, $150 \mathrm{mM} \mathrm{NaCl}, 1 \mathrm{mM}$ EGTA, $1 \mathrm{mM}$ EDTA, $1 \mathrm{mM}$ sodium orthovanadate, $1 \mathrm{mM}$ sodium $\beta$-glycerophosphate, $50 \mathrm{mM}$ sodium fluoride, $5 \mathrm{mM}$ sodium pyrophosphate, $1 \%$ Triton X-100 and $0.5 \%$ Nonidet P-40) supplemented with complete protease inhibitors (one tablet per $25 \mathrm{ml}$; Roche) and $0.1 \%$ $\beta$-mercaptoethanol (Sigma) or $50 \mathrm{mM}$ iodoacetamide (Sigma) or $2.5 \mathrm{mg} \mathrm{ml}^{-1}$ dithiobis[succinimidyl propionate] (DSP) (Thermo Scientific). Lysates were cleared and processed immediately or snap frozen in liquid nitrogen and stored at $-80^{\circ} \mathrm{C}$. The protein concentration was determined with a photo spectrometer using Bradford protein assay reagent (Pierce). For RNA isolation, cells were processed using an RNA extraction kit according to the manufacturer's instructions (Qiagen RNeasy kit). To assay luciferase reporter activity, cells were lysed in passive lysis buffer (Promega), and luciferase activity was measured on a MicroLumat plus LB 96V luminometer from Berthold Technologies. The luciferase counts were normalized to the protein concentration and averaged.

Mouse tissue isolation. Tissues from mice were frozen in liquid nitrogen and grounded with mortar and pestle. Powdered tissues were resuspended in tissue lysis buffer (10 mM Tris-HCl pH 8, $150 \mathrm{mM} \mathrm{NaCl}, 1 \mathrm{mM}$ EDTA, 1\% NP40, 0.1\% SDS and protease inhibitors) and incubated on ice for $30 \mathrm{~min}$ before centrifugation. The cleared extracts were processed as described for cell extracts.

Immunoprecipitation (IP). Cleared cell extracts were mixed with FLAG-agarose beads, HA-agarose beads, GFP-Trap agarose beads (Chromatek) or antibody-coupled protein G-sepharose beads for $2 \mathrm{~h}$ at $4{ }^{\circ} \mathrm{C}$ on a rotating platform. The flowthrough was retained for western blot analysis and the beads were washed twice in lysis buffer containing $0.4 \mathrm{M} \mathrm{NaCl}$, and twice in buffer A ( $50 \mathrm{mM}$ Tris- $\mathrm{HCl} \mathrm{pH} 7.5$, $0.1 \mathrm{mM}$ EGTA). IP samples, as well as flow-through and input samples, were reduced in SDS sample buffer $(62.5 \mathrm{mM}$ Tris- $\mathrm{HCl} \mathrm{pH} 6.8,10 \%$ (v/v) glycerol, $2 \%$ $(\mathrm{w} / \mathrm{v})$ SDS, $0.02 \%(\mathrm{w} / \mathrm{v})$ bromophenol blue, $5 \%(\mathrm{v} / \mathrm{v}) \beta$-mercaptoethanol) and heated at $95^{\circ} \mathrm{C}$ for $5 \mathrm{~min}$. Where appropriate, $\lambda$-phosphatase $(1 \mu \mathrm{M})$ was added to OTUB1 IPs resuspended in $50 \mu \mathrm{l}$ buffer $(50 \mathrm{mM}$ HEPES, $100 \mathrm{mM} \mathrm{NaCl}, 0.01 \%$ Brij$35,2 \mathrm{mM}$ dithiothreitol (DTT), $1 \mathrm{mM} \mathrm{MnCl} 2, \mathrm{pH} 7.5$ ) or whole-cell extracts and mixed at $30^{\circ} \mathrm{C}$ for $30 \mathrm{~min}$. IPs were then washed and processed as stated above.

SDS-PAGE and western blotting. Reduced protein extracts $(20 \mu \mathrm{g}$ protein unless stated otherwise) or IPs were separated on 10\% SDS-PAGE gels, 4-20\% TGX gels (Bio-Rad) or 4-12\% NuPAGE bis-tris gels (Invitrogen) by electrophoresis and transferred to polyvinylidene fluoride membranes (Millipore). Membranes were blocked in $5 \%(\mathrm{w} / \mathrm{v})$ non-fat milk in TBS-T $(50 \mathrm{mM}$ Tris- $\mathrm{HCl} \mathrm{pH} \mathrm{7.5,150} \mathrm{mM}$ $\mathrm{NaCl}, 0.2 \%$ Tween-20), incubated overnight at $4{ }^{\circ} \mathrm{C}$ in $5 \%$ milk-TBS-T or $3 \% \mathrm{BSA}-$ TBS-T with the appropriate primary antibodies followed by incubation with horseradish peroxidase (HRP)-conjugated secondary antibodies in 5\% milk-TBS-T and detection by ECL luminescence (Thermo Scientific). Full scans of key western blots are provided in Supplementary Fig. S8.

Antibodies. Anti-phospho-Ser463/465 SMAD1 (SMAD1-TP; number 9511), anti-phospho-Ser465/467 SMAD2 (SMAD2-TP; number 3101), anti-SMAD2/3 (number 3102), anti-SMAD4 (number 9515) and anti-GAPDH (number 2118) antibodies were obtained from Cell Signalling. Anti-phospho-Ser423/425 SMAD3 (SMAD3-TP; number 600-401-919) and anti-phospho-Thr179 SMAD3 (SMAD3-LP; number 600-401-C48S) were purchased from Rockland. AntiSMAD7 (number MAB2029) was from R\&D Systems, anti-tubulin from Calbiochem (number DM1A) and anti-ubiquitin from Dako (number Z0458). Anti-K48 ubiquitin (clone Apu2; number 05-1307) was from Millipore. Human recombinant SMAD1(141-268) polypeptide and full-length OTUB1 were used as immunogens in sheep to generate anti-SMAD1 and anti-OTUB1 antibodies, respectively. HRPconjugated FLAG-(number A8592), HA-(number 12013819001) and GST-(number ab3416) antibodies were from Sigma, Roche and Abcam, respectively. For western blot analysis, all primary antibodies were used at 1:1,000 dilution, except for FLAG, GST, tubulin and GAPDH antibodies, which were used at 1:5,000 dilution. HRP-coupled secondary antibodies $(1: 5,000)$ were obtained from Pierce and HRP-coupled light chain-specific secondary antibodies $(1: 10,000)$ were purchased from Jackson Immuno Research.

Peptide binding assay. Biotin-C6-RQTVTSTPCWIELHLNGPLQWLDKVLTQM GSPSVRCS-Sp-M-Sp (SMAD2-TP; $3 \mu \mathrm{g}$ ) was incubated with purified GST-OTUB1 $(5 \mu \mathrm{g})$ or pre-cleared $\mathrm{HaCaT}$ cell lysate $(150 \mu \mathrm{g}$; with or without pre-treatment with TGF $\beta(50 \mathrm{pM}, 1 \mathrm{~h})$ ) in lysis buffer for $30 \mathrm{~min}, 30^{\circ} \mathrm{C}, 900$ r.p.m. As a control, the phospho-peptide was additionally treated with $\lambda$-phosphatase $(18 \mu \mathrm{M})$ in buffer (50 mM HEPES, $100 \mathrm{mM} \mathrm{NaCl}, 0.01 \%$ Brij-35, $2 \mathrm{mM}$ DTT, $1 \mathrm{mM} \mathrm{MnCl} \mathrm{m}_{2}$ pH 7.5). Streptavidin-Sepharose High Performance beads (GE Healthcare) were equilibrated in lysis buffer and $20 \mu \mathrm{l}$ of 50/50 slurry beads were added for $15 \mathrm{~min}, 30^{\circ} \mathrm{C}, 1,300$ r.p.m. IPs were washed six times in lysis buffer with $0.4 \mathrm{M}$ $\mathrm{NaCl}$ and twice in buffer A. Proteins were resolved by SDS-PAGE and immunoblotted (IB).
Mass spectrometry. For mass spectrometric analysis, HEK293 cells expressing the protein of interest were lysed in HEPES lysis buffer ( $40 \mathrm{mM}$ HEPES pH 7.4, $120 \mathrm{mM} \mathrm{NaCl}, 1 \mathrm{mM}$ EDTA, $10 \mathrm{mM}$ sodium pyrophosphate, $50 \mathrm{mM}$ sodium fluoride, $1.5 \mathrm{mM}$ sodium orthovanadate, $1 \%$ Triton X-100) supplemented with $0.5 \mu \mathrm{M}$ microcystin-LR, $2.5 \mathrm{mg} \mathrm{ml}^{-1}$ dithiobis[succinimidyl propionate] (DSP) and complete protease inhibitors (one tablet per $25 \mathrm{ml}$ ). The lysate was incubated for $30 \mathrm{~min}$ on ice and the crosslinking reaction was quenched by the addition of Tris- $\mathrm{HCl} \mathrm{pH} 7.4$ (200 mM final). Lysates were then cleared by centrifugation (15,000 r.p.m., $30 \mathrm{~min})$ and filtration $(0.45 \mu \mathrm{m})$. Subsequently, the lysates were incubated with Protein A-agarose beads $\left(1 \mathrm{~h}\right.$ on a rotating platform at $\left.4{ }^{\circ} \mathrm{C}\right)$ to limit unspecific binding. The cleared lysate $(50 \mathrm{mg})$ was incubated with GFP-Trap beads $(50 \mu \mathrm{l})$ for $4 \mathrm{~h}$ on a rotating platform at $4^{\circ} \mathrm{C}$. The beads were washed four times in complete lysis buffer containing $0.4 \mathrm{M} \mathrm{NaCl}$ and twice in $10 \mathrm{mM}$ Tris-HCl, $\mathrm{pH} 7.4$ The beads were reconstituted in $1 \times$ LDS (Invitrogen) containing $0.1 \mathrm{M}$ DTT, incubated at $37^{\circ} \mathrm{C}$ for $1 \mathrm{~h}$ and heated at $95^{\circ} \mathrm{C}$ for $5 \mathrm{~min}$. The samples were separated by gel electrophoresis, stained with Colloidal Coomassie (Invitrogen) and prepared for mass fingerprinting. Peptide mass fingerprinting data analysis was performed using OLMAT (http://www.proteinguru.com/MassSpec/OLMAT).

Real-time qRT-PCR. $\mathrm{HaCaT}$ cells were seeded in six-well plates, transfected with siRNAs and serum starved for $16 \mathrm{~h}$ before TGF $\beta(50 \mathrm{pM})$ treatment. Complementary DNA was made from $1 \mu \mathrm{g}$ of the isolated RNA using the I-Script cDNA kit (Bio-Rad) according to the manufacturer's protocol. $\mathrm{qRT}$-PCR reactions were performed in quadruplicates according to the manufacturer's protocol in a CFX 384 Real-time System qRT-PCR machine (Bio-Rad).

All primers were designed using PerlPrimer and were purchased from Invitrogen. Primers used are as follows $\left(5^{\prime}-3^{\prime}\right)$ : OTUB1 forward: ACAGAAGATC AAGGACCTCCA, reverse: CAACTCCTTGCTGTCATCCA, FoxO4 forward: TTGGAGAACCTGGAGTGTGACA, reverse: AAGCTTCCAGGCATGACTCAG, PAI-1 forward: AGCTCCTTGTACAGATGCCG, reverse: ACAACAGGAGGAGA AACCCA, CTGF forward: GGAGATTTTGGGAGTACGG, reverse: TACCAATG ACAACGCCTCCT, GAPDH forward: TGCACCACCAACTGCTTAGC, reverse: GGCATGGACTGTGGTCATGAG, RPL13A forward: CCTGGAGGAGAAGAGG AAAGAGA, reverse: TTGAGGACCTCTGTGTATTTGTCAA.

The primer efficiency was determined and taken into account when evaluating the qRT-PCR data. The data were normalized to the geometrical mean of two housekeeping genes (GAPDH, RPL13A). The Pfaffl method ${ }^{55}$ was used to analyse the qRT-PCR data in Microsoft Excel.

Ubiquitylation/deubiquitylation assays. In-cell ubiquitylation/deubiquitylation assays were performed in HEK293 cells by co-transfecting FLAG-SMAD3, HAubiquitin and appropriate HA-OTUB1/OTUB1 mutants or NEDD4L constructs. In vitro ubiquitylation/deubiquitylation assays were performed with human recombinant E1, E2 and E3 enzymes, GST-OTUB1 or mutants and SMAD proteins (expressed and purified by DSTT, Axel Knebel or Ubiquigent). For chain cleavage assays, DUBs $\left(30 \mathrm{ng} \mathrm{ml}^{-1}\right)$ were incubated in DUB buffer $(50 \mathrm{mM}$ Tris- $\mathrm{HCl} \mathrm{pH}$ 7.5, $5 \mathrm{mM}$ DTT, $100 \mathrm{mM} \mathrm{NaCl})$ with the indicated ubiquitin chains $\left(0.13 \mu \mathrm{g} \mu \mathrm{l}^{-1}\right.$, $\mathrm{K} 48 \mathrm{ub}_{2-7}, \mathrm{~K}^{\circ} \mathrm{ub}_{2-7}$ (Boston Biochem), K48 $\mathrm{ub}_{2}$ (Axel Knebel)) on an IP shaker for $1 \mathrm{~h}$ at $30^{\circ} \mathrm{C}$.

Ubiquitylation assays of SMAD2 or FLAG-SMAD2/3/4 (immunoprecipitated from HEK293 cells treated with $50 \mathrm{pM}$ TGF $\beta$ for $1 \mathrm{~h}$ before lysis) were performed in ubiquitylation assay buffer ( $50 \mathrm{mM}$ Tris- $\mathrm{HCl}$ pH 7.5, $5 \mathrm{mM} \mathrm{MgCl} 2,2 \mu \mathrm{M}$ ATP) with $\mathrm{His}_{6}$-UBE1 $(0.1 \mu \mathrm{M})$, UBE2D1 $\left(1 \mu \mathrm{M}\right.$ or indicated concentrations), $\mathrm{His}_{6}$ NEDD4L $(1 \mu \mathrm{M})$ and ubiquitin $\left(50 \mu \mathrm{M}\right.$; Sigma) for $1 \mathrm{~h}$ at $30{ }^{\circ} \mathrm{C}$ on an IP shaker. The DUBs (30 ng $\mu \mathrm{l}^{-1}$ or at increasing concentrations $\left(8-60 \mathrm{ng} \mu \mathrm{l}^{-1}\right)$ ) were added at the start of the ubiquitylation assay (time 0 ).

In vitro DUB assays of in vivo polyubiquitylated FLAG-SMAD2/3/4 (FLAGSMAD2/3/4 were co-expressed with HA-NEDD4L and HA-ubiquitin in HEK293 cells, treated with $50 \mathrm{pM}$ TGF $\beta$ and $10 \mu \mathrm{M}$ bortezomib for $3 \mathrm{~h}$ and FLAG was immunoprecipitated) or in vitro-polyubiquitylated SMAD3 were performed with indicated DUBs in DUB buffer for $1 \mathrm{~h}$ at $30^{\circ} \mathrm{C}$ on an IP shaker. Proteins were resolved by SDS-PAGE and were immunoblotted.

For E2 ubiquitin-loading assays, reactions were performed in $25 \mu \mathrm{l}$ buffer (50 mM HEPES, $150 \mathrm{mM} \mathrm{NaCl}, 0.5 \mathrm{mM}$ TCEP) using His ${ }_{6}$-UBE1 $(0.5 \mu \mathrm{g})$, UBE2D1 $(2 \mu \mathrm{g})$, FLAG-ubiquitin ( $2 \mu \mathrm{g}$; Sigma), GST-OTUB1 $(5.5 \mu \mathrm{g}), \mathrm{His}_{6}$ NEDD4L $(1.4 \mu \mathrm{g})$, SMAD3 $(0.55 \mu \mathrm{g})$ and $2 \mu \mathrm{l} \mathrm{Mg}$-ATP solution $(2 \mathrm{mM}$ ATP, $100 \mathrm{mM} \mathrm{Mg}$-acetate). Proteins were mixed on an IP shaker before the addition of ATP for $10 \mathrm{~min}$ at $30^{\circ} \mathrm{C}$. After ATP addition, proteins were mixed for a further $10 \mathrm{~min}$ at $30^{\circ} \mathrm{C}$ before terminating the reaction with $4 \times$ LDS sample buffer. Ten microlitres of each reaction was separated by SDS-PAGE. The gels were stained with Coomassie InstantBlue (Expedeon) and imaged.

Cellular migration assay. HaCaT cells stably expressing control shRNA or OTUB1 shRNA were seeded to near-confluency into migration inserts (Ibidi) and transfected with FoxO4 or OTUB1 siRNAs, respectively. After $24 \mathrm{~h}$ the inserts were removed, the cells were serum starved for $4 \mathrm{~h}$ and stimulated with TGF $\beta(50 \mathrm{pM})$. Cellular migration was monitored and pictures were taken every $24 \mathrm{~h}$. 
Statistical analysis. All experiments have a minimum of three biological replicates. Luciferase experiments additionally have three and qRT-PCR experiments four technical repeats for each biological repeat. Data are presented as the mean with error bars indicating the s.d. Statistical significance of differences between experimental groups was assessed with Student's $t$-test. Differences in means were considered significant if $P<0.05$. Differences with $P<0.05$ are annotated as *, $P<0.01$ are annotated as ${ }^{* *}$ and $P<0.001$ are annotated as ${ }^{* * *}$. All western blots shown are representative of biological replicates.

\section{References}

1. Akhurst, R. J. \& Hata, A. Targeting the TGFbeta signalling pathway in disease. Nat. Rev. Drug Discov. 11, 790-811 (2012).

2. Inman, G. J. Switching TGFbeta from a tumor suppressor to a tumor promoter. Curr. Opin. Genet. Dev. 21, 93-99 (2011).

3. Massague, J. TGFbeta in cancer. Cell 134, 215-230 (2008).

4. Drabsch, Y. \& ten Dijke, P. TGF- $\beta$ signalling and its role in cancer progression and metastasis. Cancer Metastasis Rev. 31, 553-568 (2012).

5. Shi, Y. \& Massague, J. Mechanisms of TGF- $\beta$ signaling from cell membrane to the nucleus. Cell 113, 685-700 (2003).

6. Feng, X. H. \& Derynck, R. Specificity and versatility in tgf-beta signaling through Smads. Annu. Rev. Cell Dev. Biol. 21, 659-693 (2005).

7. Moustakas, A. \& Heldin, C. H. The regulation of TGF $\beta$ signal transduction. Development 136, 3699-3714 (2009).

8. Massague, J. TGF $\beta$ signalling in context. Nat. Rev. Mol. Cell Biol. 13, 616-630 (2012).

9. Lonn, P., Moren, A., Raja, E., Dahl, M. \& Moustakas, A. Regulating the stability of TGFbeta receptors and Smads. Cell Res. 19, 21-35 (2009).

10. Al-Salihi, M. A., Herhaus, L. \& Sapkota, G. P. Regulation of the transforming growth factor beta pathway by reversible ubiquitylation. Open Biol. 2, 120082 (2012).

11. Bruce, D. L. \& Sapkota, G. P. Phosphatases in SMAD regulation. FEBS Lett. 586, 1897-1905 (2012).

12. De Boeck, M. \& ten Dijke, P. Key role for ubiquitin protein modification in TGFbeta signal transduction. Ups. J. Med. Sci. 117, 153-165 (2012).

13. Dupont, S., Inui, M. \& Newfeld, S. J. Regulation of TGF-beta signal transduction by mono- and deubiquitylation of Smads. FEBS Lett. 586, 1913-1920 (2012).

14. Gao, S. et al. Ubiquitin ligase Nedd $4 \mathrm{~L}$ targets activated Smad2/3 to limit TGF- $\beta$ signaling. Mol. Cell 36, 457-468 (2009).

15. Inoue, Y. \& Imamura, T. Regulation of TGF- $\beta$ family signaling by E3 ubiquitin ligases. Cancer Sci. 99, 2107-2112 (2008).

16. Sapkota, G., Alarcon, C., Spagnoli, F. M., Brivanlou, A. H. \& Massague, J. Balancing BMP signaling through integrated inputs into the Smad1 linker. Mol. Cell 25, 441-454 (2007).

17. Aragon, E. et al. A Smad action turnover switch operated by WW domain readers of a phosphoserine code. Genes Dev. 25, 1275-1288 (2011).

18. Komander, D., Clague, M. J. \& Urbe, S. Breaking the chains: structure and function of the deubiquitinases. Nat. Rev. Mol. Cell Biol. 10, 550-563 (2009).

19. Balakirev, M. Y., Tcherniuk, S. O., Jaquinod, M. \& Chroboczek, J. Otubains: a new family of cysteine proteases in the ubiquitin pathway. EMBO Rep. 4, 517-522 (2003).

20. Edelmann, M. J. et al. Structural basis and specificity of human otubain 1-mediated deubiquitination. Biochem. J. 418, 379-390 (2009).

21. Messick, T. E. et al. Structural basis for ubiquitin recognition by the Otu1 ovarian tumor domain protein. J. Biol. Chem. 283, 11038-11049 (2008).

22. Wang, T. et al. Evidence for bidentate substrate binding as the basis for the K48 linkage specificity of otubain 1. J. Mol. Biol. 386, 1011-1023 (2009).

23. Li, S. et al. Regulation of virus-triggered signaling by OTUB1- and OTUB2mediated deubiquitination of TRAF3 and TRAF6. J. Biol. Chem. 285, 4291-4297 (2010)

24. Stanisic, V., Malovannaya, A., Qin, J., Lonard, D. M. \& O’Malley, B. W. OTU Domain-containing ubiquitin aldehyde-binding protein 1 (OTUB1) deubiquitinates estrogen receptor (ER) alpha and affects ERalpha transcriptional activity. J. Biol. Chem. 284, 16135-16145 (2009).

25. Sun, X. X., Challagundla, K. B. \& Dai, M. S. Positive regulation of p53 stability and activity by the deubiquitinating enzyme Otubain 1. EMBO J. 31, 576-592 (2012).

26. Goncharov, T. et al. OTUB1 modulates c-IAP1 stability to regulate signalling pathways. EMBO J. 32, 1103-1114 (2013).

27. Soares, L. et al. Two isoforms of otubain 1 regulate T cell anergy via GRAIL. Nat. Immunol. 5, 45-54 (2004).

28. Edelmann, M. J., Kramer, H. B., Altun, M. \& Kessler, B. M. Post-translational modification of the deubiquitinating enzyme otubain 1 modulates active RhoA levels and susceptibility to Yersinia invasion. FEBS J. 277, 2515-2530 (2010).

29. Juang, Y. C. et al. OTUB1 Co-opts Lys48-linked ubiquitin recognition to suppress E2 enzyme function. Mol. Cell 45, 384-397 (2012).

30. Nakada, S. et al. Non-canonical inhibition of DNA damage-dependent ubiquitination by OTUB1. Nature 466, 941-946 (2010).
31. Sato, Y. et al. Molecular basis of Lys-63-linked polyubiquitination inhibition by the interaction between human deubiquitinating enzyme OTUB1 and ubiquitin-conjugating enzyme UBC13. J. Biol. Chem. 287, 25860-25868 (2012).

32. Wiener, R., Zhang, X., Wang, T. \& Wolberger, C. The mechanism of OTUB1mediated inhibition of ubiquitination. Nature 483, 618-622 (2012).

33. Al-Salihi, M. A., Herhaus, L., Macartney, T. \& Sapkota, G. P. USP11 augments TGFbeta signalling by deubiquitylating ALK5. Open Biol. 2, 120063 (2012).

34. Zhang, Y., Feng, X., We, R. \& Derynck, R. Receptor-associated Mad homologues synergize as effectors of the TGF- $\beta$ response. Nature 383, 168-172 (1996).

35. Lagna, G., Hata, A., Hemmati-Brivanlou, A. \& Massague, J. Partnership between DPC4 and SMAD proteins in TGF- $\beta$ signalling pathways. Nature 383, 832-836 (1996).

36. He, W. et al. Hematopoiesis controlled by distinct TIF1gamma and Smad4 branches of the TGFbeta pathway. Cell 125, 929-941 (2006).

37. Luo, K. et al. The Ski oncoprotein interacts with the Smad proteins to repress TGFbeta signaling. Genes Dev. 13, 2196-2206 (1999).

38. Osada, S., Ohmori, S. Y. \& Taira, M. XMAN1, an inner nuclear membrane protein, antagonizes BMP signaling by interacting with Smad1 in Xenopus embryos. Development 130, 1783-1794 (2003).

39. Pan, D. et al. The integral inner nuclear membrane protein MAN1 physically interacts with the R-Smad proteins to repress signaling by the transforming growth factor-\{beta\} superfamily of cytokines. J. Biol. Chem. 280, 15992-16001 (2005).

40. Calonge, M. J. \& Massague, J. Smad4/DPC4 silencing and hyperactive Ras jointly disrupt transforming growth factor-beta antiproliferative responses in colon cancer cells. J. Biol. Chem. 274, 33637-33643 (1999).

41. Teicher, B. A., Ara, G., Herbst, R., Palombella, V. J. \& Adams, J. The proteasome inhibitor PS-341 in cancer therapy. Clin. Cancer Res. 5, 2638-2645 (1999).

42. Kuratomi, G. et al. NEDD4-2 (neural precursor cell expressed, developmentally down-regulated 4-2) negatively regulates TGF- $\beta$ (transforming growth factorbeta) signalling by inducing ubiquitin-mediated degradation of Smad2 and TGF-beta type I receptor. Biochem. J. 386, 461-470 (2005).

43. Heldin, C. H., Landstrom, M. \& Moustakas, A. Mechanism of TGF-beta signaling to growth arrest, apoptosis, and epithelial-mesenchymal transition. Curr. Opin. Cell Biol. 21, 166-176 (2009).

44. Medici, D., Potenta, S. \& Kalluri, R. Transforming growth factor-beta2 promotes Snail-mediated endothelial-mesenchymal transition through convergence of Smad-dependent and Smad-independent signalling. Biochem. J. 437, 515-520 (2011).

45. Akhurst, R. J. \& Derynck, R. TGF-beta signaling in cancer-a double-edged sword. Trends Cell Biol. 11, S44-S51 (2001).

46. Thrower, J. S., Hoffman, L., Rechsteiner, M. \& Pickart, C. M. Recognition of the polyubiquitin proteolytic signal. Embo J. 19, 94-102 (2000).

47. Igarashi, A., Okochi, H., Bradham, D. M. \& Grotendorst, G. R. Regulation of connective tissue growth factor gene expression in human skin fibroblasts and during wound repair. Mol. Biol. Cell 4, 637-645 (1993).

48. Lund, L. R. et al. Transforming growth factor-beta is a strong and fast acting positive regulator of the level of type-1 plasminogen activator inhibitor mRNA in WI-38 human lung fibroblasts. EMBO J. 6, 1281-1286 (1987).

49. Pattison, M. J., MacKenzie, K. F. \& Arthur, J. S. C. Inhibition of JAKs in macrophages increases lipopolysaccharide-induced cytokine production by blocking IL-10-mediated feedback. J. Immun. 189, 2784-2792 (2012).

50. Wiggin, G. R. et al. MSK1 and MSK2 are required for the mitogen- and stressinduced phosphorylation of CREB and ATF1 in fibroblasts. Mol. Cell Biol. 22, 2871-2881 (2002).

51. Vogt, J., Traynor, R. \& Sapkota, G. P. The specificities of small molecule inhibitors of the TGFss and BMP pathways. Cell Signal. 23, 1831-1842 (2011).

52. Durocher, Y., Perret, S. \& Kamen, A. High-level and high-throughput recombinant protein production by transient transfection of suspensiongrowing human 293-EBNA1 cells. Nucleic Acids Res. 30, E9 (2002).

53. Bruce, D. L., Macartney, T., Yong, W., Shou, W. \& Sapkota, G. P. Protein phosphatase 5 modulates SMAD3 function in the transforming growth factorbeta pathway. Cell Signal. 24, 1999-2006 (2012).

54. Sapkota, G. et al. Dephosphorylation of the linker regions of Smad1 and Smad $2 / 3$ by small C-terminal domain phosphatases has distinct outcomes for bone morphogenetic protein and transforming growth factor-beta pathways. J. Biol. Chem. 281, 40412-40419 (2006).

55. Pfaffl, M. W. A new mathematical model for relative quantification in real-time RT-PCR. Nucleic Acids Res. 29, e45 (2001).

\section{Acknowledgements}

We thank Dave Campbell, Robert Gourlay, Joby Varghese, Nick Morrice and Matthias Trost for help with mass spectrometry. We thank Kirsten McLeod and Janis Stark for help with tissue culture, the staff at the Sequencing Service (School of Life Sciences, University of Dundee, Scotland) for DNA sequencing and the protein 
production teams at the Division of Signal Transduction Therapy (DSTT; University of Dundee) coordinated by Hilary McLauchlan and James Hastie for the expression and purification of proteins and antibodies. We also thank Alex Knebel and Richard Ewan for OTUB1 expression and purification. We thank Janis Vogt, David Bruce, Gareth Inman, Christophe Lachaud, Michael Pattison, Paul Davies, Noor Esoof, Dennis Castor, Craig MacKay, Arno Alpi, Kevin Dingwell (NIMR) and Jim Smith (NIMR) for various reagents and helpful discussions. We thank the Medical Research Council and the Pharmaceutical Companies supporting the DSTT (AstraZeneca, Boehringer-Ingelheim, GlaxoSmithKline, Merck-Serono, Pfizer and Johnson \& Johnson) for financial support.

\section{Author contributions}

L.H. designed the experiments and analysed the data. L.H. performed most of the experiments. M.A.-S. performed experiments for Fig. 6c. T.M. and S.W. performed the cloning. G.P.S. conceived and designed the project. L.H. and G.P.S. wrote the manuscript.

\section{Additional information}

Supplementary Information accompanies this paper at http://www.nature.com/ naturecommunications

Competing financial interests: The authors declare no competing financial interests.

Reprints and permission information is available online at http://npg.nature.com/ reprintsandpermissions/

How to cite this article: Herhaus, L. et al. OTUB1 enhances TGF $\beta$ signalling by inhibiting the ubiquitylation and degradation of active SMAD2/3. Nat. Commun. 4:2519 doi: 10.1038/ncomms3519 (2013).

(c) (i) (2) This work is licensed under a Creative Commons Attributionco NG SA NonCommercial-ShareAlike 3.0 Unported License. To view a copy of this license, visit http://creativecommons.org/licenses/by-nc-sa/3.0/ 